\title{
Mammalian diseases of phosphatidylinositol transfer proteins and their homologs
}

\author{
Aaron H Nile ${ }^{1, *}$, Vytas A Bankaitis ${ }^{\dagger, 1}$, and Aby Grabon ${ }^{1, *}$ \\ ${ }^{1}$ Department of Cell \& Developmental Biology, Lineberger Comprehensive Cancer Center School \\ of Medicine, University of North Carolina at Chapel Hill, Chapel Hill, NC 27599-27090, USA
}

\section{Abstract}

Inositol and phosphoinositide signaling pathways represent major regulatory systems in eukaryotes. The physiological importance of these pathways is amply demonstrated by the variety of diseases that involve derangements in individual steps in inositide and phosphoinositide production and degradation. These diseases include numerous cancers, lipodystrophies and neurological syndromes. Phosphatidylinositol transfer proteins (PITPs) are emerging as fascinating regulators of phosphoinositide metabolism. Recent advances identify PITPs (and PITP-like proteins) to be coincidence detectors, which spatially and temporally coordinate the activities of diverse aspects of the cellular lipid metabolome with phosphoinositide signaling. These insights are providing new ideas regarding mechanisms of inherited mammalian diseases associated with derangements in the activities of PITPs and PITP-like proteins.

\section{Keywords}

inositol signaling; lipid metabolism; phosphoinositide; phosphatidylinositol transfer protein; vertebrate disease

\begin{abstract}
Inherited mammalian diseases ultimately reduce themselves to derangements in activities of proteins. For enzymes, deficiencies in activity are understood in terms of the substrates and products of defined chemical reactions. For scaffolding or structural proteins, compositions of protein complexes describe the defects. However, in other cases, the proteins (or protein domains) of interest resist simple functional definitions. Such molecules are often understudied for these reasons, and phosphatidylinositol transfer proteins (PITPs) fall into this category. The goal of this review is to summarize the important physiological functions executed by these proteins with an emphasis on the inherited diseases associated with altered activities of individual PITPs or PITP-like proteins. With regard to more mechanistic questions, we discuss recent advances that provide new insights into how these proteins operate at the molecular level. Of particular emphasis are emerging concepts regarding how PITPs integrate phosphoinositide signaling with diverse aspects of intracellular lipid metabolism. These insights provoke fresh perspectives from which to view potential mechanisms of mammalian PITP diseases.
\end{abstract}

\footnotetext{
(C) 2010 Future Medicine Ltd

${ }^{\dagger}$ Author for correspondence: Tel.: +1 919962 9870, Fax: +1 919966 1856, vytas@ med.unc.edu.

* These authors contributed equally to the work

Financial \& competing interests disclosure

The authors have no other relevant affiliations or financial involvement with any organization or entity with a financial interest in or financial conflict with the subject matter or materials discussed in the manuscript apart from those disclosed.

No writing assistance was utilized in the production of this manuscript.
} 


\section{Inositol signaling}

The involvement of phosphorylated forms of $\mathrm{p}$-myo-inositol (Ins-phosphates) and phosphatidylinositol (phosphoinositides) in eukaryotic signal transduction is well documented [1]. Indeed, the breadth of inositide and phosphatidylinositol (PtdIns)-based signaling has inspired some to anoint Ins as evolution's favorite molecule [2]. This is not an idle proclamation given the diversity of phosphorylated products that can be generated from Ins-containing compounds. For example, yeast generate five phosphoinositides (PtdIns-3-P, PtdIns-4-P, PtdIns-5-P, PtdIns-4,5- $\mathrm{P}_{2}$ and PtdIns-3,5- $\mathrm{P}_{2}$ ), while higher eukaryotes produce seven (the five listed for yeast plus PtdIns-3,4-P $\mathrm{P}_{2}$ and PtdIns-3,4,5 $\mathrm{P}_{3}$ ). The case for Insphosphates is more impressive. As each position of the six-member Ins ring can be phosphorylated (and in at least several cases pyrophosphorylated), the cabal of possible soluble Ins-phosphate species is immense (63 + Ins for monophosphates and $728+$ Ins if one imposes a limit of only two phosphates per Ins-OH). These statistics identify the versatility of Ins as a six-bit chip where specific signaling information is encoded by a unique combination of positionally specific phosphorylations on the Ins ring. The Insphosphate chemical code is subsequently interpreted by proteins, which have the appropriate Ins-phosphate binding specificities.

Use of Ins as a signaling scaffold, either in the form of a soluble Ins-phosphate or a membrane-incorporated phosphoinositide, requires a fine coordination between biosynthetic activities (PtdIns-kinases) and degradative processes (catalyzed by phospholipases and phosphoinositide phosphatases). Comprehensive reviews focusing on the metabolism of Insphosphates and phosphoinositides treat these issues in detail, and the reader is referred to them [3-5]. In this regard, the physiological importance of Ins and PtdIns metabolism is obvious. Defects in the enzymes that directly catalyze specific biosynthetic or degradative reactions in Ins-phosphate or phosphoinositide metabolic pathways result in a variety of inherited human diseases [6,7]. The landscape assumes even greater complexity when issues of spatial or temporal control of phosphoinositide production and degradation (i.e., issues critical to biological regulation of Ins-phosphate and phosphoinositide signaling) are considered. In this article, we limit the discussion to the production arm of phosphoinositide signaling.

Present discussions of the roles for phosphoinositides in cell regulation focus on, first, the function of these lipids as metabolic reservoirs for second messengers (e.g., diacylglycerol and soluble Ins-phosphates), and second, their involvement in the formation of membranebinding platforms for specific proteins [8-10]. Regarding the latter context, the ability of a mammalian cell to produce seven chemically distinct phosphoinositides allows for creation of a diverse set of binding platforms. The chemical heterogeneity of phosphoinositides is in turn interpreted by protein-binding motifs such as pleckstrin homology $(\mathrm{PH})$ domains, Fab1, YOTB, VAC1 and EEA1 (FYVE) domains, PX domains, and even basic patches on protein surfaces that execute phosphoinositide binding by purely electrostatic mechanisms [8-10].

Discussions of phosphoinositide signaling are dominated by product-centric models that fail to capture important dynamics that accompany production of these lipids. These discussions also do not adequately describe the consequences these mechanisms have with regard to functional diversification of phosphoinositide signaling. The principle message to be delivered in this article is that we do not yet understand important aspects of how lipid signaling is regulated in eukaryotic cells, nor do we understand how the larger lipid metabolome is integrated with phosphoinositide signaling. An emerging concept that bears on this theme that is a specific phosphoinositide generated by a specific lipid kinase can nonetheless have multiple biological outcomes in a single-cell eukaryote [11-13]. Thus, biological outcome is not solely determined by the chemical nature of the phosphoinositide, 
nor is it determined by the PtdIns kinase that produced it. Rather, biological outcome tracks with nonenzymatic proteins that stimulate PtdIns kinase activities. These regulatory proteins are the PtdIns/phosphatidylcholine (PtdCho)-transfer proteins (PITPs) and the data suggest that these PITPs 'instruct' physiological outcomes for PtdIns kinase activities [11-13]. PITP-like protein domains hold similar potential for providing such instructive functions, and these domains are found in intriguing contexts. The importance of PITPs and PITPdomain proteins in eukaryotic cell biology and physiology is amply demonstrated by the mammalian diseases associated with derangements in the function of such proteins. Herein, we review the PITPs and mammalian diseases of PITP-like protein dysfunction.

\section{Operational definitions for the PITPs}

\section{Sec14-like \& START-like PITPs}

All eukaryotes express PITPs. The so-called 'classical' PITPs (a purely historical definition) mobilize PtdIns and PtdCho transfer between membranes in vitro. These PITPs bind PtdIns and PtdCho in a mutually exclusive manner. PtdIns is the preferred binding substrate, and the rate of PtdIns-transfer is some 20-fold greater than that for PtdCho [14]. This preference reflects the greater affinity of PITP for PtdIns relative to PtdCho. At present, PITPs are most often interpreted to function as lipid carriers that supply PtdIns, synthesized in endoplasmic reticulum membranes, to membranes that are low in PtdIns (e.g., the plasma membrane) yet execute an active phosphoinositide cycle [15]. The 'nonclassical' PITPs are designated as such because these retain the ability to bind or transfer PtdIns, but do not conserve PtdChobinding or transfer activity $[11,16,17]$. The nonclassical PITPs, which, ironically, almost certainly outnumber the classical versions, provide interesting cases for how the diversity in lipid binding by PITPs and PITP-like proteins translates to the great diversity in biological outcome for phosphoinositide signaling (see later).

Phosphatidylinositol transfer proteins are highly conserved. The conservation of PITPs breaks down into two distinct branches based on their structural folds, which are the Sec14like PITPs and the START-like (StAR-related lipid transfer domain) PITPs. To date, all START-like PITPs studied are classical PITPs, while Sec14-like proteins include both classical and nonclassical varieties. As described in detail below, the Sec14 and START folds are unrelated, although these do share some general properties. Whether Sec14-like and START-like PITPs evolutionarily converge on common functional mechanisms, or whether their shared transfer activities are purely coincidental, remains to be determined.

Fungi, plants, metazoans and apicomplexan parasites are rich in Sec14-like proteins, and these constitute an ancient and uniquely eukaryotic protein superfamily. The founding member of this PITP class is yeast Sec14 [17-19]. As detailed later, the Sec14 superfamily counts the mammalian retinaldehyde binding proteins, domains of Rho-GEF proteins, the neurofibromin Ras-GAPs and plant phosphoinositide binding proteins among more than 1500 of its members. Even simple eukaryotes such as yeast express multiple Sec14-like proteins (Saccharomyces cerevisiae expresses six), while Drosophila melanogaster, Caenorhabditis elegans, mice, humans and plants (e.g., Arabidopsis thaliana) each express more than 20 Sec14-domain proteins. By contrast, the START-like PITP family is a rather sparse one $[17,20]$ and is further subdivided into type 1 and 2 PITPs. The soluble STARTlike PITPs (type 1 PITPs) have an approximate molecular weight of $35 \mathrm{kDa}$ and are homologous to each other. The type 2 proteins are larger constructions with a domain homologous to the entire type 1 START-like PITP sequence appended to the N-termini of large membrane-associated modules. The START-like PITP family is not expanded to a large degree from flies (two type 1 PITPs and one type 2 PITP) to humans (three type 1 PITPs and two type 2 PITPs). The type 2 PITPs exhibit complex modular arrangements, but the PITP domain is the essential component of at least one of these proteins - the 
approximately 900 -amino acid Drosophila type 2 PITP RdgB. This protein is required for the fly photoresponse - a high-capacity phosphoinositide signaling system. However, the 280 -residue PITP domain of RdgB (comprises only 25\% of the total RdgB protein sequence) is both necessary and sufficient for rescue of the retinal degeneration associated with $\mathrm{RdgB}$ inactivation, and for restoration of a seemingly wild-type photoresponse in flies lacking the full-length protein [21]. This review focuses on type 1 PITPs because these are better represented in models for mammalian disease.

\section{The slippery faces of lipid transfer activities}

As PITPs are not enzymes, translation of PITP-associated lipid exchange activities to biochemical or biological mechanisms is difficult. While discussions of biological mechanisms for PITP function remain anchored to the historical concept that PITPs are bona fide carrier proteins that deliver lipid from one intracellular membrane system to another (Figure 1A), such arguments are inherently circular. That is, PITPs are defined on the basis of an operational transfer assay of uncertain functional significance, and the transfer activity is subsequently featured as the central cellular activity executed by the PITP. Arguments that directly translate PITP in vitro transfer activities to facilitated mobilization of lipid between intracellular membranes in vivo are wrapped in important biological assumptions. One central assumption made in such transfer models is that lipid synthesis is restricted to a few intracellular compartments. As our understanding of cellular lipid biosynthetic capabilities grows, this assumption is coming under increasing fire.

Despite the general acceptance of lipid transfer mechanisms, there is little direct evidence to support simple transfer models for any individual PITP. This evidentiary gap reflects the difficulties in experimentally testing transfer models in physiologically relevant settings. Are there other perspectives from which to view the PITP or lipid transfer problem? Insights culled from studies on PITPs, particularly PITPs of the Sec14 superfamily, do indeed suggest new and detailed mechanistic possibilities. The available evidence is most consistent with Sec14, and other Sec14-like proteins, functioning as 'primed' lipid biosensors that couple binding of lipids other than PtdIns (sensor function) to a PtdIns-presentation activity (Figure 1B). The PtdIns-presentation function potentiates the PtdIns-kinase activity by making PtdIns a better substrate for the enzyme. Thus, Sec14-like PITPs are engaged in the action of small machines, or nanoreactors, where metabolic and signaling reactions are integrated and the products are generated in a spatially and temporally appropriate manner. We define a minimal nanoreactor as a functional interaction between a phospholipid-bound PITP and a PtdIns kinase. 'Nanoreactor' models do not describe PITPs as transorganelle lipid carriers, and offer new perspectives on how to interpret functions of PITP-like modules in multidomain proteins. The Sec14 paradigm provides new ideas from which to view mechanisms of PITP function and recent evidence suggests that these new concepts might extend to type 1 PITPs.

\section{Sec14-like PITPs as molecules}

\section{Sec14 \& integration of Ptdlns/phosphatidylcholine metabolic signals}

Sec14, the major yeast PITP, is required for membrane trafficking through the trans-Golgi network (TGN) or endosomal system. It acts in a retrograde 'endosome to TGN' trafficking capacity, and is essential for yeast cell viability [13,17,22]. 'Bypass Sec14' mutations, that permit yeast viability in the absence of the normally essential Sec14, provide unique avenues for diagnosing how Sec14 translates its PtdIns/PtdCho-transfer activities to biological function [23-28]. The 'bypass Sec14' mutants reveal a remarkably intimate coupling between the cellular requirement for Sec14 function and activity of the CDPcholine pathway for PtdCho biosynthesis. That is, inactivation of the CDP-choline pathway 
obviates the cellular Sec14 requirement $[24,25]$. These studies also show that yeast mutants deranged for phospholipid biosynthesis, such that PtdIns is the major membrane phospholipid ( $40 \mathrm{~mol} \%$ compared with $20 \mathrm{~mol} \%$ for wild-type yeast and $5 \mathrm{~mol} \%$ for mammalian cells; mol\% represents a unit describing the fractional contribution of a specific phospholipid species to the total number of phospholipid molecules in, in this case, the cell), still require Sec14 for cell viability. A PtdIns surfeit of this magnitude should present a condition where PITP-driven PtdIns-supply requirements are no longer necessary; however, the Sec14 requirement for cell viability and TGN/endosomal function stands. These various data are difficult to reconcile with PtdIns- and PtdCho-transfer models for Sec14 [24,25].

Rather, 'bypass Sec14' mutants identify Sec14 as an essential integrator required for proper coordination of a specific arm of PtdCho-metabolism with phosphoinositide synthesis. This integration is essential for membrane trafficking through the TGN/endosomal system $[12,13,17,24-28]$.

\section{Anatomy of phospholipid exchange by Sec14-like PITPs}

All ideas regarding mechanisms of PITP function assign an important role for the phospholipid-exchange activities of these proteins. These remarkable activities are sustained by thermal energy alone and require no additional cofactors. What are the mechanics of the phospholipid exchange reaction from the perspective of the PITP and from the perspective of phospholipid ligand? Crystallographic studies demonstrate the Sec14-domain (smart00516) to constitute an approximately 280-amino acid, two-lobed, globular structure that encases a large hydrophobic cavity, which defines the phospholipid-binding pocket $[12,29,30]$. Electron paramagnetic resonance measurements report that the hydrophobicity parameters of the pocket are such that this cavity, from the perspective of the phospholipid ligand, offers an environment that is similar to that provided by a membrane leaflet. Thus, incorporation of a phospholipid from a membrane into the Sec14 interior, and vice versa, is primarily driven by partitioning of a phospholipid between two chemically equivalent environments [31]. How the phospholipid is brought to the point where such a partitioning choice is available remains unclear.

Access to the binding pocket is gated by a helical substructure whose configuration is flipped open in apo-Sec14 conformers that occur when Sec14 is docked onto membrane surfaces (Figure 2A) [12,29,32]. The helical gate is closed in holo-Sec14 conformers (Figure 2B), and these represent solution configurations for Sec14::PtdIns and Sec14::PtdCho complexes. The transitions between the open and closed conformers that accompany phospholipid binding and release on membrane surfaces are dominated by an $18 \AA$ displacement of the helical gate (Figure 2). Helical gate dynamics are controlled by a compact 'gating module' that regulates an extensive H-bond network through which conformational information is transduced to the helical gate upon membrane binding [32].

\section{The core engineering of a Sec14 nanoreactor}

How does Sec14 use its PtdIns- and PtdCho-transfer activities to integrate PtdCho metabolism with phosphoinositide synthesis? The solution to this problem is encoded in the way Sec14 binds to its phospholipid ligands. The most remarkable feature of Sec14 (and Sfh1) is the striking difference in the binding poses of PtdIns and PtdCho upon incorporation into the hydrophobic pocket (Figure 3). While the acyl chain regions of each phospholipid occupy overlapping physical space, the respective headgroups are stabilized by distant regions of the hydrophobic pocket. This curious and unexpected engineering for how Sec14 binds distinct phospholipid headgroups is of functional significance as heterotypic phospholipid exchange capability must be housed within individual protein molecules in order for Sec14 to potentiate PtdIns 4-OH kinase activities in vivo [12,13]. In principle, Sec14 employs a coincidence-detection strategy that integrates PtdCho metabolic 
information with the action of PtdIns 4-OH kinases - in other words, Sec14 employs its heterotypic PtdIns- or PtdCho-exchange activities to sense (bind) local PtdCho and so prime a 'PtdIns-presentation' unit, or nanoreactor, that stimulates PtdIns 4-OH kinases. The necessity for such a complex program stems from the biological inadequacy of PtdIns 4-OH kinases as interfacial enzymes when confronted with PtdIns substrates incorporated into genuine membrane bilayers [12,13].

\section{Primed Ptdlns-presentation models versus lipid transfer models}

The concept that Sec14 employs heterotypic exchange in a concerted phospholipid sensing/ presentation cycle portrays the associated in vitro PtdIns- or PtdCho-transfer activities in a very different light than do lipid transfer models. An attractive aspect of presentation/ nanoreactor models are these frame-specific and experimentally testable hypotheses. Several are presented here for discussion. While the questions are framed in the context of Sec14, each of these ideas generates questions that generally pertain to functional interpretations of PITPs and other lipid transfer proteins.

Presentation models do not demand physical transfer of lipids from one intracellular destination to the other - it is the cycle that is the key. How many average exchange cycles does Sec14 complete during its membrane dwell time? Presentation models embrace the possibility that many such cycles are executed by Sec14 during each short episode where the protein associates with a membrane, while transfer models describe a scenario where there is one exchange cycle per membrane association event.

Is physical disengagement of Sec14 from membranes required for execution of biological function? The simplest transfer models demand this be the case, although membrane 'contact site' models leave open the possibility that a membrane-bound Sec14 could still retain biological function. Space limitations prohibit detailed discussions of 'contact-site' models but the concept is reviewed elsewhere [33,34], and readers are referred to these for further information. Presentation models easily accommodate scenarios where Sec14 (or other LTP domains) are biologically functional as membrane-bound multidomain molecules.

Is a complete cycle of exchange obligatory for function, or are abortive exchanges productive? Transfer models demand completion of a pick-up and delivery cycle - not so for presentation models. The 'presentation' concept describes a trapping of PtdIns molecules in a transitory state - one where the PtdIns is neither fully membrane- nor protein-incorporated - and is therefore particularly vulnerable to modification by PtdIns 4-OH kinases. Such a mechanism posits that an invading PtdIns molecule is prevented from fully incorporating into the Sec14 hydrophobic pocket by a leaving PtdCho. Such a frustrated PtdIns molecule can be marked by a PtdIns 4-OH kinase without the PtdIns ever having fully incorporated itself into the Sec14 hydrophobic pocket. Interestingly, PtdCho enters and exits the hydrophobic pocket much more slowly than PtdIns. This raises the possibility that multiple rounds of abortive PtdIns incorporation may occur per single PtdCho egress event, thereby providing a physical picture of priming. A formal corollary to this hypothesis is that an appropriate covalent adduct of PtdCho, or any other suitable steric obstacle, within the Sec14 hydrophobic pocket might still be compatible with the capability of Sec14 to stimulate PtdIns 4-OH kinase activity, even though this arrangement is physically incompatible with lipid exchange.

What trajectories do lipid molecules follow during entry or exit from the Sec14 hydrophobic pocket? Do PtdIns and PtdCho share similar trajectories (e.g., same entry and exit portals), or do these trace different paths? Reductionist questions of this sort are not particularly important for understanding interorganelle lipid transfer mechanisms. However, solutions to these questions are central to an understanding of how presentation/nanoreactor mechanisms 
work because heterotypic phospholipid trajectories define the operative anatomy of the presentation process.

Finally, how are Sec14 'sensing' territories defined? This question relates to how spatial regulation of phosphoinositide synthesis is controlled. We define a sensing territory as that area on a given membrane where the PITP is executing biologically productive heterotypic phospholipid exchange reactions (i.e., result in enhanced phosphoinositide synthesis). For membrane-tethered versions of Sec14-like PITPs (see later), the spatial restriction of the tethering (by accessory membrane-binding domains, membrane binding via protein-protein or protein-lipid interactions intrinsic to the Sec14 domain), determines the sensing territory. However, the cytosolic Sec14 potentiates the activities of distinct PtdIns 4-OH kinases that reside in distinct intracellular compartments within the same cell. How is this accomplished for such a nomadic PITP? Transient interactions of Sec14 with a guiding platform (protein or lipid) might contribute at some level. The PtdIns 4-OH kinase itself is an obvious candidate for such a landmark. However, the ability of the structurally unrelated vertebrate type 1 PITPs to act as functional Sec14 surrogates in yeast argues against privileged Sec14PtdIns 4-OH kinase interactions [35-37]. As discussed in detail elsewhere [13], more stochastic arrangements can still be biologically productive (i.e., result in enhanced synthesis of phosphoinositide).

\section{A PITP-centric strategy for linking lipid metabolism to phosphoinositide signaling}

The Sec14 fold is an evolutionarily ancient and versatile one, conserved from single-cell eukaryotes to humans. Its expansion throughout the eukaryotic kingdom reflects an impressive diversification of the unit to bind a wide variety of lipids and lipophilic molecules. Primary sequence comparisons identify well-conserved crystal structure-based PtdIns-binding signatures in many of these proteins; however, PtdCho-binding signatures are not extensively conserved $[12,13]$. These binding signatures, or bar codes, represent the translation of 3D structural information into a 2D primary sequence read-out. The PtdInsbinding bar code is depicted in Figure 4. It is an attractive proposition that Sec14 superfamily proteins couple metabolism of a diverse set of lipids or lipophilic molecules (i.e., of Sec14-protein ligands) with phosphoinositide synthesis, thereby coordinating disparate arms of the lipid metabolome with common phosphoinositide signaling pathways. These models might generally apply to PITPs and PITP-domain proteins. We highlight these new perspectives in this article.

\section{Mammalian Sec14-domain protein disorders}

Mammals employ the versatile Sec14 fold in diverse ways - in some cases as standalone domains or, more frequently, as modules that contribute to more complex arrangements in multidomain proteins. Owing to the sheer scope of the Sec14 superfamily, only a limited sampling of proteins can be summarized here. We restrict attention to those Sec14-like proteins whose dysfunction is related to inherited mammalian disease.

\section{Standalone Sec14-like proteins \& disease}

The standalone Sec14-like proteins regulate a variety of cellular events, including the visual cycle, vitamin E homeostasis, apoptosis and membrane trafficking. Individual derangements in these standalone Sec14-like proteins primarily manifest themselves as neurological disorders. Some outstanding examples are summarized later. In some cases, the identities of the ligands that occupy the hydrophobic pocket are known. For others, no ligand that incorporates into the protein interior is known. However, many Sec14-like proteins exhibit a recognizable PtdIns-binding barcode [12]. Whether these proteins do indeed bind to PtdIns, 
and whether these are capable of channeling PtdIns to phosphoinositide synthesis, raises interesting questions for study.

a-tocopherol transfer protein \& vitamin E status-Ataxia with vitamin E deficiency (AVED) is an autosomal recessive, progressive neurodegenerative disorder caused by deficiencies in the Sec14-like $\alpha$-tocopherol binding protein ( $\alpha$ TTP) [38]. This human disease is hallmarked by low vitamin E levels, and manifests itself through hyporeflexia, ataxia, muscle weakness, dementia, visual field contraction and even complete blindness, and cardiac arrhythmias [39]. Treatment for AVED involves high doses of orally administered vitamin E (1200-1500 mg/day), which restores vitamin $\mathrm{E}$ to normal circulating levels of $0.5-2.0 \mathrm{mg} / \mathrm{dl}$. Indeed, if administered prior to extensive progression of disease, either preor postsymptomatic delivery of vitamin E can effectively reverse, or entirely prevent, AVED [40]. Mice engineered for $\alpha$ TTP deficiency similarly exhibit low levels of circulating vitamin E, and present late-onset neurological deficits [41-43].

At least 25 mutations have been described in the 278-amino acid, $\alpha$ TTP structural gene (TTPA). These fall into two clinical categories: those resulting in severe AVED with early onset, and those characterized by milder AVED with late onset [44]. Several mutations in conserved residues (e.g., R59W, E141K and R221W) compromises $\alpha$-tocopherol ( $\alpha$-TOH) binding or transferring and result in severe AVED [45]. By contrast, the R192H, H101Q and A120T missense substitutions involve partially conserved residues, do not strongly compromise $\alpha$-TOH binding/transfer and result in mild AVED [46,47].

It is generally accepted that $\alpha \mathrm{TTP}$ is the master regulator of plasma vitamin E levels. In comparative studies, $\alpha$ TTP preferentially binds and transfers $\alpha$-TOH between membranes in vitro relative to other tocopherols [48-50]. It is thought that $\alpha$ TTP employs such a transfer activity to channel $\alpha-\mathrm{TOH}$ to a secretory, rather than a degradatory, fate [51,52]. Some models suggest that $\alpha$ TTP does so by mediating direct transport of $\alpha$-TOH from endosomes to the plasma membrane for incorporation into VLDLs in an ABCA1 transporter-dependent hepatic secretory pathway [53]. Consistent with this view, $\alpha$ TTP expression enhances $\alpha$ TOH secretion in cultured hepatocytes $[47,54,55]$. The Sec14 nanoreactor concept suggests an alternative model - $\alpha$ TTP may link heterotypic $\alpha$-TOH/PtdIns binding or exchange to generation of a phosphoinositide pool dedicated to biogenesis of $\alpha$-TOH-rich exocytic vesicles. This model predicts that compromise of the PtdIns-binding barcode in $\alpha$ TTP will inactivate the protein. In this regard, the $\mathrm{R} 221 \mathrm{~W}$ missense substitution, which results in severe AVED, directly alters the PtdIns-binding barcode. This position corresponds to Sec14 residue K239. This residue helps coordinate binding of the phosphate moiety through which the Ins headgroup is esterified to the glycerol backbone. Substitutions at this position specifically compromise PtdIns binding by Sec14 [12,30]. Similarly, the R192H AVEDassociated missense substitution in $\alpha$ TTP corresponds to Sec14 amino acid G210 - a residue positioned adjacent to core elements of the PtdIns-binding barcode.

Caytaxin \& cerebellar ataxia-Cayman-type cerebellar ataxia is a rare autosomal recessive disorder whose incidence is limited to an isolated population on the Grand Cayman Island resulting from defects in the brain-specific, Sec14-like presynaptic protein termed caytaxin. Clinical manifestations include cerebellar hypoplasia, psychomotor retardation, hypotonia from birth, prominent nonprogressive cerebellar dysfunctions that manifest through intention tremors, dysarthric speech and a wide-base ataxic gait. This disease is distinguished from other ataxias by the presence of nystagmus and the lack of retinal defects [56,57]. Although lipid ligand(s) for caytaxin are unknown, structural modeling suggests PtdIns lipids are tenable candidates [56,58] - a concept fortified by a recognizable structural barcode for PtdIns binding [12]. Analyses of Cayman-type ataxic individuals reveal two polymorphisms. One disrupts an exon-intron boundary, leading to 
truncation of much of the protein, and an S301R missense mutation [56]. Our analyses project S301 to fall into the helical gate region of the caytaxin Sec14 fold, suggesting gate dynamics that regulate transitions between open and closed caytaxin conformers might be compromised. An interesting question for future address is whether compromise of the putative caytaxin PtdIns-binding barcode inactivates the protein.

Much of what is known about caytaxin is derived from the study of rodent models. Caytaxin derangements result in a spectrum of motor malfunctions/dystonia in the jittery (ji), hesitant $\left(\mathrm{ji}^{\text {hes }}\right)$, sidewinder (jiswd ) and wobbly mice [301], and the well-characterized dystonic (dt) rat [59]. A battery of electrophysiological and biochemical studies define the olivocerebellar pathway, particularly in the response of Purkinje cells to climbing fiber projections, as the point of functional abnormality in the dt rat [60]. The dt rat cerebellar cortex exhibits altered transcript levels for signaling pathway components that regulate cell-surface signaling, calcium homeostasis, extracellular matrix and PtdIns signaling [58]. Upregulation of caytaxin in human prefrontal cortex is also associated with altered calcium homeostasis and immune system imbalances in schizophrenia [61]. Interestingly, while dt rats normally die by postnatal day 40 , cerebellectomy rescues both ataxia and viability, suggesting that aberrant cerebellar signaling lies at the root of the observed dysfunctions [62-64].

Caytaxin physically interacts with a number of proteins, including the E3 ubiquitin ligase CHIP [65], and peptidyl-prolyl isomerase during neuronal differentiation [66]. These associations suggest caytaxin function may be regulated by its binding to these proteins. Furthermore, the caytaxin Sec14 domain binds to the kidney-type glutaminase - an enzyme that converts glutamine to the abundant neurotransmitter glutamate. Caytaxin overexpression results in the translocation of the enzyme from the cell body to neurite terminals, and reduces steady-state glutamate levels by inhibiting glutaminase. On this basis, it is speculated that caytaxin deficiency-associated glutamate elevation underlies the clinical manifestations of cayman-type cerebellar ataxia [65]. Moreover, overexpression of either caytaxin or its Sec14-domain alone results in the elongation of processes in MCF-7 cells, as is the case with overexpression of the caytaxin homologue BNIP-2 [67,68]. Finally, caytaxin also scaffolds kinesin light chain 1 in cultured hippocampal cells, thereby facilitating transport of vesicle cargo [68].

\section{Cellular retinaldehyde binding protein \& the vertebrate visual cycle-In} vertebrates, light absorption by opsin results in photoisomerization of 11-cis-retinaldehyde (11-cis-RAL) to all-trans-retinaldehyde (all-trans-RAL). Thus, 11-cis-RAL regeneration is essential for a sustained vertebrate visual cycle. Detailed description of the vertebrate visual cycle is beyond the scope of this review; and the reader is referred to detailed reviews on the subject $[69,70]$. An important component of 11-cis-RAL regeneration is the Sec14-like cellular retinal-binding protein (CRALBP) 1. Multiple pathologies are associated with CRALBP1 dysfunction, including: retinitis pigmentosa, fundus albipunctatus, Newfoundland rod/cone dystrophy and Bothnia dystrophy. It is suggested that all of these disorders are manifestations of retinitis punctata albescens (a flecked retinal dystrophy characterized by early-onset night blindness, uniform white-yellow spots across the fundus and the progression of macula and retina atrophy resulting in blindness), which is also a manifestation of CRALBP1 insufficiencies [70,71]. Moreover, mice deficient in CRALBP1 manifest large reductions in rates of rhodopsin regeneration, 11-cis-RAL production, and dark adaptation after illumination. Unlike the case in humans, photoreceptor degeneration is not observed [72].

Sec14-like cellular retinal-binding protein 1 is a soluble protein, primarily expressed in retinal pigment epithelium cells and in Müller cells, but not in their adjacent photoreceptors [70]. In retinal pigment epithelium cells, CRALBP1 directly bind the 11-cis-retinol (11-cis- 
ROL) formed after the isomerization of all-trans-retinyl ester, or from activated 11-cisretinyl esters used as a storage mechanism [73]. CRALBP1 functions primarily to regulate esterification of 11-cis-ROL [73], and to act as a carrier molecule to assist in the oxidation of 11-cis-ROL to 11-cis-RAL in the vertebrate visual cycle [74]. CRALBP1 associates with 11-cis-retinol-dehydrogenase (RDH5) in a ternary complex that involves interaction with ezrin, actin and the PDZ domain of EPB-50. In this fashion, CRALBP1 is hypothesized to metabolically channel 11-cis-ROL to RDH5 for oxidation [75]. CRALBP1 binds to acidic phospholipids, and this binding promotes release of bound 11-cis-retinal [76]. It is not yet clear whether 11-cis-retinal release is mediated by competition for an overlapping binding site within the CRALBP1 hydrophobic pocket (i.e., in effect a Sec14-like heterotypic lipid exchange reaction) or whether acidic phospholipid interactions with the protein surface evoke conformational changes that eject 11-cis-retinal.

Several naturally occurring mutations in CRALBP1 compromise retinoid binding (e.g., R151Q and M226K), or enhance its binding (e.g., R234W) [77,78]. In this regard, the autosomal recessive Bothnia dystrophy presents an interesting case. Pathologies include night blindness in early childhood and progressive macular/peripheral retinal degeneration $[79,80]$. Bothnia dystrophy occurs in one per 3500 births worldwide, with increased incidence in northern Sweden, primarily as a result of inheritance of the R234W and M226K variants [79]. The crystal structures of 11-cis-RAL-bound CRALBP1 and the R234W mutant were recently solved [81]. These studies reveal that R234W further stabilizes bound 11-cis-RAL by increasing packing interactions within the binding cavity. In addition, R234 resides in a conserved basic cleft of CRALBP1. R234 corresponds to Sec14 residue R208, which helps coordinate PtdIns binding by Sec14 and is a component of the Sec14 structural barcode for PtdIns binding [12,30].

Although discussions of CRALBP1 are dominated by its involvement in the vertebrate visual cycle, elevated CRALBP1 levels are associated with altered calcium homeostasis and immune system imbalances in schizophrenia [61]. CRALBP1 may also represent a human autoimmune uveitis autoantigen [82], and its status may affect ethanol preference in mice [83].

\section{Multidomain Sec14-like proteins \& disease}

The Sec14 superfamily is too large to cover in one review. The distant members of the superfamily, the BNIP proteins, which primarily function in apoptosis, are reviewed elsewhere [84,85], and will not be emphasized here. A partial list of multidomain Sec14-like proteins not emphasized in this article is provided in Table 1. Instead, we focus on a set of examples relevant to human disease.

Small GTPases of the Rho/Rac/Cdc42 families regulate a number of cellular activities such as migration, cytoskeleton dynamics, cell cycle progression, gene expression, cell adhesion and others [86]. These do so by functioning in binary switch mode between GTP- (active) and GDP-(inactive) bound states. Modular proteins with Sec14-domains include a number of regulators of small GTPase signaling; in other words, guanine nucleotide exchange proteins (GEFs) and GTPase-activating proteins (GAPs). Sec14-like domains are also associated with other enzymatic activities such as protein kinases and protein tyrosine phosphatases. In these multidomain protein contexts, Sec14 domains are posited to function as nanoreactors that stimulate 'on demand' phosphoinositide synthesis in the immediate vicinity of the particular catalytic domain of the protein, thereby effecting an efficient regulation of protein enzymatic activity [13]. The spectrum of diseases caused by derangements in Sec14-like proteins includes various cancers, neurological disorders, developmental and trafficking defects. Some outstanding examples are summarized below and the relevant Sec14-domains often present recognizable PtdIns-binding barcodes. 
RhoGEFs with Sec14 domains-The Dbl family of RhoGEFs is defined by an approximate 200-residue Dbl homology (DH) domain positioned adjacent to a C-terminal of an approximate 100-residue plekstrin homology $(\mathrm{PH})$ domain. Of the approximately $70 \mathrm{Dbl}-$ family RhoGEFs, four (Dbl, Dbs/Ost, Duo/Kalirin and Trio) exhibit Sec14-domains $[84,86,87]$. All four RhoGEFs are expressed as multiple slice variants, not all of which harbor a Sec14 domain, thereby offering mechanisms for differentially regulating the functional properties and subcellular localization of individual isoforms [88-91].

Dbl-Dbl, the founding member of the Dbl family of RhoGEFs, is represented by at least four splice variants, three of which contain a Sec14 domain. This domain regulates Dbl localization and GEF activities [92], and binds PtdIns-3-P, PtdIns-4-P and PtdIns-5-P in vitro [89]. Oncogenic forms of Dbl exist that exclude the N-terminal 496 residues of the protein, thereby truncating the Sec14 domain and several spectrin repeats $[87,93]$. These oncogenic forms of $\mathrm{Dbl}$ influence cell migration, cell polarity and vascularization of epithelial tissue in murine lens [94]. Dbl-null mice are rather normal phenotypically, although these do present measurable defects in dendrite elongation [95].

Kalirin/Duo-Kalirin/Duo is a neuronal RhoGEF represented by at least eleven forms, six of which contain a Sec14 domain (Kalirin-SOLO, 4, 7, 8, 9 and 12) [96-98]. Full-length kalirin (Kalirin-12) is a complex protein that exhibits a Sec14-like domain, nine spectrin-like repeats, two DH, two PH, two SH3, one immunoglobin, one fibronectin III and one Ser/Thr protein kinase-like domain (Figure 5) [99]. The isolated Kalirin Sec14-domain is reported to bind phosphoinositides based on crude lipid blot assays [98]. Kalirin nullizygous mice show cognitive and working memory deficiencies associated with reduced neuronal spine densities and abnormal spine morphologies [100]. These neuronal morphology defects are also manifested in ex vivo culture [101]. In addition, Kalirin is implicated as a genetic risk factor for ischemic stroke [102], coronary artery disease [103], Alzheimer's disease [104] and schizophrenia $[100,105]$.

The predominant Kalirin, Kalirin-7, is an important regulator of dendritic spine development and functional plasticity $[106,107]$. NMDA receptor activation in pyramidal neurons induces a CaMkII-dependent phosphorylation of Kalirin-7 on its Sec14-domain. This phosphorylation stimulates Kalirin-7 GEF activity, and elicits enlargement of neuronal spines via enhanced activation of Rac1 [108]. Moreover, the Kalirin-7 Sec14-domain may also interact with GB $\gamma$ subunits of heterotrimeric G-proteins [109]. Although it is clear that the Sec14 domain is important for Kalirin-7 function, the mechanisms for how the Sec14 domain interfaces with other Kalirin-7 domains is not understood. As the Sec14 domain is implicated as a negative regulators of other Dbl family members [88], and phosphorylation of the Sec14 domain promotes GEF activity, a negative regulatory role is a distinct possibility [108]. Whether lipid binding is involved in such a circuit remains to be determined. Moreover, variants produced from an alternative translation start site truncate the Sec14 domain and the first four spectrin repeats $(\Delta$-Kalirin-7) exhibit distinct properties with regard to regulation of endocytosis, solubility, oligomerization state, cytoskeleton binding and subcellular localization [98].

Trio-Trio contains a Sec14 domain and eight to nine spectrin repeats linked to two DH domains, two PH domains, two SH3 modules, one immunoglobin domain and one Ser/Thr kinase catalytic domain (Figure 5) [87,110]. Trio is represented by at least six isoforms, five of which contain a Sec14 domain [91]. Trio nullizygous mice fail in embryonic development with deranged organization of neural tissues and defects in fetal skeletal muscle [111]. Recent studies implicate Trio in netrin-1/deleted in colorectal cancer (DCC)-dependent axon guidance through its ability to activate Rac1 [110], and its expression is associated with invasive tumor growth and rapid tumor cell proliferation in bladder cancer [112]. Moreover, 
genome-wide association studies also link Trio expression to esophageal squamous cell carcinoma [113]. The short Solo/Trio8 isoform (contains the Sec14 domain), which is primarily expressed in Purkinje cells, localizes to endosomes where it activates Rho GTPases and promotes neurite elongation in developing Purkinje cells. The Sec14 domain is suggested to contribute to endosomal localization of this isoform [114]. Human Trio also potentiates the nerve growth factor pathway for RhoG- and Rac1-dependent neurite outgrowth in PC12 cells. However, the Sec14 domain is dispensable for the neuritepromoting activity of Trio [115].

Dbs-The Dbs/Ost RhoGEF is a proto-oncogene that modulates cell motility in humanderived breast epithelial cells via activation of Cdc42 and Rac1 [116]. The protein consists of an N-terminal Sec14 domain, two spectrin repeats, a DH domain, a PH domain and an SH3 domain (Figure 5) [88]. The purified Dbs Sec14 domain binds a variety of phosphoinositides in crude lipid blot assays [88], and the Sec14 domain is responsible for directing Dbs subcellular localization so that it can interact with its primary substrate Cdc42 [89]. Sec14-domain activities are not simple as this module also inhibits Dbs transforming activity by interacting with the PH domain and regulating subcellular localization [88]. Whether this Sec14/PH-domain interaction is regulated by lipid binding remains to be determined.

RhoGAPs-The RhoGAP family is populous - counting in excess of 70 members. Of those, p50RhoGAP/Cdc42GAP and BPGAP1 are similar proteins that exhibit N-terminal Sec14-domains appended to RhoGAP domains by proline-rich linker domains (Figure 5) [117].

CDC42GAP/p50RhoGAP—Mice deficient for Cdc42GAP exhibit multiple premature aging defects including reduction in body mass, loss of subdermal adipose, muscular atrophy, osteoporosis and delayed wound healing [118], and present enhanced rates of c-Jun $\mathrm{N}$-terminal kinase (JNK)-mediated basal apoptosis [119]. As may be expected, Cdc42GAPdeficient murine embryonic fibroblasts display elevated Cdc42 activity, and these cells are prone to spontaneous formation of filipodia with defects in directional migration [120]. Cdc42GAP derangements are implicated in human disorders such as Waldenstrom macroglobulinemia [121] and human chronic myeloid leukemia [122]. CDC42GAP is also suggested to be a counter-regulator of tubule formation, forecasting a role in angiogenesis [123].

The Sec14-like domain is responsible for localization of Cdc42GAP to endosomes as evidenced by the fact that missense substitutions in the presumptive Sec14-like lipid-binding pocket result in Cdc42GAP mislocalization. In that regard, Cdc42GAP interacts with the Rab11 GTPase, suggesting a link between Rab and Rho GTPases and endosome dynamics [124]. Cdc42GAP exists in an autoinhibited state that is controlled in part by intermolecular interactions between amino acids 1-48 and 169-197, which reside in the Sec14-like domain. Interaction with the prenyl group of small GTPases promotes the release of autoinhibition [125].

Neurofibromin RasGAPs-Neurofibromin (NF)-1 encodes for a 2818-residue RasGAP that is homologous to the yeast RasGAPs, Ira1 and Ira2 [126,127]. Defects in NF-1 result in the progressive, autosomal dominant disorder, neurofibromatosis type 1, affecting one in 3500 individuals worldwide. The disease manifests through multiple brown skin macules (café-au-lait spots), intertriginous freckling, iris hamartoma (Lisch nodules) and learning disabilities. NF1 patients are also at a higher risk for optical gliomas and neurofibromas $[128,129]$. NF-1 primarily regulates p21-Ras-GTP levels, thereby modulating downstream 
cascades including Ras-MAPK and Akt-mTOR pathways. Loss of NF-1 activity deregulates of these proproliferative pathways and inhibits apoptosis [130].

NF-1 has three discrete domains: the RasGAP catalytic module [131], the Sec14-like domain [132] and a PH domain [127]. Several other domains have recently been defined largely on the basis of bioinformatic analyses (Figure 5) [133]. A number of missense substitutions elicit NF1 loss-of-function phenotypes without destabilizing the protein $[134,135]$. Several of these substitutions map to the Sec14 domain, thereby demonstrating the functional importance of this domain for NF-1 biological activity. A series of these disease-associated mutations affect residues that either comprise, or flank, the hinge domain of Sec14-like proteins. These substitutions likely interfere with conformational transitions of the helix, which gates the hydrophobic pocket [136]. In addition, a tandem repeat mutation identified in a neurofibromatosis patient with Noonan's disease duplicates a linker region between the NF1 Sec14 and PH domains, indicating that interdomain communications between the Sec14, PH and RasGAP domains are required for operative NF1 activity [127].

Sec14-like protein tyrosine phosphatase-A Sec14 module is incorporated into the $68 \mathrm{kDa}$, cytoplasmic, protein-tyrosine phosphatase MEG2/PTPN9 [137-139]. In vitro experiments suggest that PTP-MEG2 binds PtdIns(3,5) $\mathrm{P}_{2}, \operatorname{PtdIns}(4,5) \mathrm{P}_{2}, \operatorname{PtdIns}(3,4,5) \mathrm{P}_{3}$ and phosphatidylserine $[137,140]$. Thus, interaction of the Sec14 domain with lipids may control both MEG2 localization and phosphatase activity. Murine MEG2 is highly expressed in the brain, liver, kidneys and testes. Mice deficient for MEG2 exhibit embryonic lethality with a penetrance of more than $90 \%$ with hemorrhage, neural tube defects, decreased size, immunodeficiency and abnormal bone development. It is suggested that many of these dysfunctions result from defects in secretory processes [141]. MEG2 targets the cytoplasmic face of secretory vesicles in a Sec14 domain-dependent manner, where it promotes vesicle fusion by dephosphorylating (and activating) the N-ethylmaleimide-sensitive factor essential for resolving cis-SNARE-pins [142]. While missense substitutions projected to compromise PtdIns binding do not prevent MEG2 association with vesicles, these substitutions do inactivate the protein for stimulating vesicular fusion $[137,143]$. The available data suggest the MEG2 Sec14 domain executes functions in addition to membrane targeting.

\section{Type 1 START-like PITPs as molecules}

As is the case for Sec14-like PITPs, the origins of START-like PITPs are rooted deep in eukaryotic evolution. Database searches identify candidate type 1 PITP-like proteins in protists with highly streamlined genomes (e.g., Giardia, Enterocytozoon and Encephalitozoon). As we highlight PITPs in the context of mammalian disease in this review, we focus primarily on what we know about the mammalian versions of these proteins. There are three mammalian START-like type 1 PITPs (PITP $\alpha, \operatorname{RdgB} \beta$ and PITP $\beta$ ). Essentially nothing is known about $\operatorname{RdgB} \beta$ and we will ignore it for the remainder of this review. Instead, we focus on the homologous PITP $\alpha$ and PITP $\beta$ - both of which are classical PITPs. These proteins are encoded by distinct genes, yet share $77 \%$ identity at the primary sequence level. PITP $\beta$ is expressed as two splice variants (termed canonical and alternative on a historical basis), which differ only in the extreme C-terminal primary sequence of the protein $[36,144,145]$. Zebrafish (Danio rerio) also express a mammalian-like cohort of type 1 PITPs with the addition of a unique PITP $\beta$-like version designated PITP $\gamma$. The type 1 PITP roster extends to interesting details of type 1 PITP diversity - in other words, zebrafish execute precisely the same exon-skipping splicing event as do mammals in generating the canonical and alternative PITP $\beta$ splice variants [37].

Crystal structures for both PITP $\alpha$ and PITP $\beta$ are available, and multiple structural models for PITP $\alpha$ have been solved (Figure 6). These include high resolution structures for PtdCho- 
bound and phospholipid-free forms [20,146], and a lower resolution structure for the PtdInsbound form [147]. As previously indicated, type 1 PITPs are structurally unrelated to Sec14like PITPs and are characterized by a START structural fold that forms a single large lipidbinding cavity. Unlike the case for Sec14-like PITPs, PtdIns and PtdCho assume very similar poses within the type 1 PITP lipid-binding cavity (Figure 6). The type 1 PITP strategy for phospholipid binding suggests these proteins may not operate in a Sec14-like nanoreactor/PtdIns-presentation mode. However, genetic data identify residues Ser25 and Pro78 as being specifically required for PtdIns-binding/transfer by PITP $\alpha$ [20] - even though neither residue uniquely contacts PtdIns, or influences other residues that do so, in the holo-PITP $\alpha$ structure. The existence of such enigmatic ligand-specific binding/transfer mutants suggests that PtdCho and PtdIns trajectories during lipid exchange are different in type 1 PITPs, but ultimately converge on similar poses in the closed conformer. Indeed, type 1 PITPs rescue both cell viability and phosphoinositide production in yeast devoid of Sec14. As yeast membranes are rich in PtdIns (20 mol\%), these data indicate type 1 PITPs can function in a Sec14-like nanoreactor/PtdIns-presentation mode [35,36]. Whether these do so in the PtdIns-poor mammalian cell (PtdIns represents $5 \mathrm{~mol} \%$ of bulk phospholipid) is difficult to demonstrate, yet structural studies suggest that the apo-PITP $\alpha$ conformer displays an open channel, which provides access to the headgroup binding region. This channel provides a path via which a lipid kinase could potentially access a PITP-bound PtdIns headgroup [146]. Such a mechanism requires productive PITP-PtdIns kinase interactions to occur during the interfacial lipid exchange reaction. This concept is consistent with nanoreactor/PtdIns-presentation modes of action.

The structural studies also suggest how type 1 PITPs interact with membrane surfaces. Type 1 PITPs present a loop with adjacent Trp residues ( $\operatorname{Trp}_{203}$ and $\operatorname{Trp}_{204}$ in PITP $\alpha$ ) and it is reported that compromise of this Trp-Trp motif inactivates the PITP - presumably by compromising PITP interaction with membranes [147]. This is a controversial issue as other studies, while demonstrating a requirement for this motif in the more stable association of PITP $\beta$ with Golgi membranes in vivo, nonetheless demonstrate the motif is neither important for the types of transient membrane interactions that accompany lipid exchange reactions nor for biological function in a vertebrate context [37,144].

\section{Cellular functions}

Despite the similarity between PITP $\alpha$ and PITP $\beta$, the proteins exhibit important differences, including PITP $\alpha$ being localized to the cytosol/nucleus while PITP $\beta$ targets the TGN; PITP $\beta$ is able to bind/transfer the ceramide-based phospholipid (PL) sphingomyelin, in addition to the glycerol-PLs PtdIns and PtdCho, while PITP $\alpha$ only binds/transfers PtdIns and PtdCho. From a functional perspective, PITP $\beta$ appears to execute important housekeeping function(s) in the face of robust PITP $\alpha$ expression [148], while PITP $\alpha$ is not essential for cell viability. As discussed in detail below, PITP $\alpha$ nullizygosity results in neonatal lethality, even though normal levels of PITP $\beta$ are expressed in the nullizygotes.

Remarkably little is known about the cellular functions of type 1 PITPs. Data from permeabilized cell systems report PITP $\alpha$ stimulates $\mathrm{Ca}^{2+}$-activated secretory granule exocytosis [149], secretory vesicle and immature granule budding from hepatocyte and neuroendocrine TGN [150] and plasma membrane receptor/G-protein-coupled phosphoinositide hydrolysis by phospholipase C (PLC) [151]. PITP $\alpha$ requirements for agonist-stimulated phosphoinositide synthesis are recorded whether signaling occurs via receptor or nonreceptor tyrosine kinases, or through PLC $\beta$ or PLC $\gamma 1$ [152,153]. Using a more physiological system, silencing experiments suggest a cellular role for mammalian PITP $\beta$ in regulating nuclear envelope morphology and retrograde membrane trafficking from cis-Golgi membranes to the endoplasmic reticulum [154]. In the following section, we review functional studies of vertebrate type 1 PITPs with murine models as primary focus. 
Both the congruence and the dissonance between cellular studies and animal studies of type 1 PITPs are discussed.

\section{Vertebrate models for type 1 PITP-associated disease}

Precisely how type 1 PITP biochemical properties translate to biological activity of the individual proteins remains to be determined. The $\mathrm{R} \operatorname{dgB} \beta$ remains uncharacterized, and only recently have insights in vertebrate PITP $\beta$ function been forthcoming $[37,154]$. However, it is clear that PITP $\alpha$, at least, is essential for the viability of vertebrate organisms including mammals. Our understanding of the physiological consequences that accompany impaired PITP $\alpha$ functionality derive from analyses of a series of mouse lines with graded reductions in PITP $\alpha$ activity. Hypomorphic lines include the vibrator homozygous mice $(v b / v b)$ and $v b /$ null heterozygous mice [155-157], which express 20 and $10 \%$ of wild-type levels of wildtype PITP $\alpha$, respectively. The $v b$ allele is the result of a serendipitous insertion of an IAP retrotransposon into an intronic region of the pitp $\alpha$ structural gene, thereby reducing the efficiency with which the cognate pre-mRNA is processed $[155,156]$.

There are currently two categories of what are operationally considered to represent pitp $\alpha$ null alleles. One is an engineered deletion that eliminates two exons encoding essential functional elements of the protein. The second is an insertion of a recombinant retrotransposon, which harbors splice-trap activity and interrupts PITP $\alpha$ mRNA translation without deleting any portion of the structural gene. Mice homozygous for either the deletion allele or the splice-trap insertion fail to produce detectable amounts of PITP $\alpha$ protein and exhibit indistinguishable phenotypes [158]. Most of the detailed characterizations executed to date involve mice homozygous for the deletion allele.

\section{PITPa \& neurological disease}

PITP $\alpha$ is produced in most (if not all) cells, but it is particularly highly expressed in brain and cerebellum. In the adult rat, PITP $\alpha$ is produced most robustly in cerebellar Purkinje neurons and granule cells [159-161]. Consistent with these expression data, murine model systems report an important role for PITP $\alpha$ in maintaining integrity of the spinocerebellar system. PITP $\alpha$ null $\left(p i t p \alpha^{0 / 0}\right)$ and hypomorphic mice exhibit striking neurological defects the severities of which are proportional to the level of PITP $\alpha$ expressed $[155,157,158]$.

The $v b$ mouse line takes its name from the rapid whole-body tremor observed in $v b / v b$ homozygotes that reflects a progressive, and ultimately fatal, neurodegenerative disease. Genetic modifiers strongly affect the lifespan of $v b / v b$ homozygotes. In the inbred C57/B6 background, these hypomorphs live for 31-35 days after birth while in outbred, or even other inbred, backgrounds lifespans of up to 6 months are recorded [155]. One such genetic modifier operates at the level of improving 'read-through' of the IAP element that defines the $v b$ insertion mutation, thereby elevating both the levels of mature PITP $\alpha$ mRNA and wild-type protein produced. The net result is that the lifespan is increased [162].

By contrast, $p i t p \alpha^{0 / 0}$ mice are born at the expected Mendelian frequencies but usually expire within several days of birth. In rare cases, the pitp ${ }^{0 / 0}$ homozygotes can persist for 10-13 days after birth. The inability of the nullizygotes to thrive is hallmarked by obvious tremor and impaired motor capacity [158]. Unlike the case of $v b / v b$ mice, the abbreviated lifespan of pitp $\alpha^{0 / 0}$ homozygotes is independent of genetic background and is accompanied by two additional signature pathologies - hypoglycemia and intestinal chylomicron retention disease (CRD) [157,158]. These syndromes are addressed in subsequent sections and, as discussed later, contribute to the rate of onset of neurological disease in PITP $\alpha$-deficient animals. 
The neurodegenerative disease course of the $v b$ mouse is classified into three phases. Phase I describes the 'true vibrator' phenotype defined by fine, high-frequency postural tremors that become apparent approximately 15 days after the birth of $p i t p \alpha^{v b / v b}$ homozygotes. Phase I postural tremors are reminiscent of the enhanced physiological tremors encountered in clinical settings $[155,163]$. While the etiology of enhanced physiological tremors in humans is not known, such tremors often present as a symptom of hyperthyroidism or metabolic dysfunction such as hypoglycemia and liver disease. In that regard, $p i t p \alpha^{0 / 0}$ mice also display hepatic steatosis and severe hypoglycemia (see below; [158]).

PITP $\alpha$ null mice do not present Phase I phenotypes. Instead, Phase II symptoms are detected from the outset, indicating neuronal damage even at the earliest stages of postnatal life $[155,157,158]$. Phase II is marked by ataxia and action tremors. The coarse intention tremor is superimposed on the animal's voluntary movements. These neurological symptoms are distinct, and not simply progressive, from Phase I symptoms. Furthermore, Phase II presents with clear anatomical signs of degeneration. Neurons in the lumbar and cervical spinal cord and in the cerebellum are vacuolated, aponecrotic and display distended endoplasmic reticulum (ER; see later). In genetic backgrounds where $v b$ mice are reasonably long lived (5-6 months), the disease progresses to a severe cerebellar atrophy [155]. For these reasons, Phase II defines the 'degenerative' phase. In the inbred C57/B6 background, Phase II persists until hours before the animal perishes. The basic presentation of Phase II disease resembles the symptoms associated with clinical cases of stroke, inherited neurodegenerative disorders and multiple sclerosis [164-166].

The terminal stages of PITP $\alpha$ insufficiency define Phase III disease characterized by loss of consciousness, decreased motor tone and fasciculations, and a progressive ascending motor paralysis that ultimately leads to asphyxiation. For C57/B6 animals, Phase III signals imminent death (within hours) and appears at approximately 31-33 days postnatal in the case of $v b / v b$ homozygotes.

\section{Anatomy of neurodegenerative disease in PITPa-deficient mice}

PITP $\alpha$ null mice exhibit robust inf lammation and demyelination in the spinal cord [158]. There are clear reductions in white matter in the cervical, thoracic and lumbar spinal cord, and damaged neurons are observed at the white and gray matter interface. Inflammation is evident over the entire length of the spinal cord, but is most striking in the ventral horn, in other words, where motor neuron cell bodies are located. Many of the neuronal cell bodies in the ventral horn present the vacuolation and low cytoplasmic content typical of aponecrosis - a form of cell death associated with critically low cellular energy charge. Indeed, measurements of ATP:ADP ratios indicate that $p i t p \alpha^{0 / 0}$ cerebellum and liver are under significant energy stress while the brain does not. Consistent with the widespread aponecrosis occurring in those regions, mast cells and macrophages infiltrate into the perivascular matrix and vessels, as well as the perivascular tissue, indicating breach of blood-brain barrier integrity. Extensive defects in myelination are obvious in both white and gray matter regions of dorsal spinal columns, and are accompanied by axonal swelling and neuropil degeneration. Another striking property of $p i t p \alpha^{0 / 0}$ brain is the reactive gliosis evident throughout the cerebellum - the organ is inundated with activated microglia. Again, vacuolations of smooth ER are prevalent in cerebellar neurons of pitp ${ }^{0 / 0}$ mice [158]. The fulminating spinocerebellar inflammatory disease indicates regulated exocytic pathways associated with the activities of inflammatory cells (e.g., mast cells) are not strongly compromised in the null animal. The results from mouse studies are incongruent with those obtained from permeabilized cell system, which suggest that PITP $\alpha$ is required for such regulated exocytic events $[149,158]$. These data are consistent with the report that $p^{i t p} \alpha^{0 / 0}$ embryonic stem cells differentiated ex vivo produce mast cells capable fully of executing the agonist-stimulated compound exocytosis that is a distinguishing property of these 
inflammatory cells [148]. Synaptic performance is also unperturbed in pitpa $\alpha^{0 / 0}$ neurons under multiple testing regimes [158]. This is a surprising result given that the synaptic vesicle cycle is a high-capacity phosphoinositide-utilizing system [4].

It is difficult to interpret whether the spinocerebellar degeneration observed in pitp $\alpha^{0 / 0}$ mice is a primary phenotype or a secondary consequence of the glucose homeostatic and CRD defects that define major phenotypes of the null condition. The substantially postnatal development of the cerebellum dictates a robust proliferative program for cerebellar neurons. Engagement of such a vigorous cell growth program, in the face of severe hypoglycemic circumstances, must soon come to a critical point where proliferation can no longer be sustained in an inadequate physiological environment. Such a catastrophic developmental failure is expected to result in manifest necrosis and induction of a fulminating inflammatory response. These predictions are fulfilled by the cerebellar inflammatory disease of PITP $\alpha$-deficient mice. With regard to the spinal cord, affected motor neurons are extraordinarily large cells, and it is likely these are especially sensitive to environmental insult. That the spinocerebellar degeneration is, at least in part, caused by a hostile physiological environment is further indicated by demonstrations that $p i t p \alpha^{0 / 0}$ cerebellar granule cells, and dorsal root ganglia from spinal cord, are not intrinsically fragile when cultured ex vivo. Moreover, titration experiments indicate these $\mathrm{pitp}^{0 / 0}$ neurons are not overly sensitive to reduced trophic factor availability relative to wild-type neurons [158].

To determine the degree of interdependence among the phenotypes observed in $p i t p \alpha^{0 / 0}$ animals, Alb et al. generated an allelic series of mice in which levels of wild-type PITP $\alpha$ protein are graded across a broad functional range. These studies establish that the threshold levels of PITP $\alpha$ activity sufficient to relieve CRD and hypoglycemia remain inadequate for sparing spinocerebellar degeneration; although onset of neurodegenerative disease is significantly delayed in the absence of hypoglycemia and CRD [157]. The collective data indicate that spinocerebellar disease is an intrinsic pathology of PITP $\alpha$-deficient mice.

\section{Cell nonautonomous mechanisms for PITPa-dependent neuroprotection}

The neurodegenerative pathologies associated with PITP $\alpha$ insufficiencies suggest a prosurvival/antiapoptotic role for PITP $\alpha$ at the cellular level. Such an activity can be formally executed in a cell nonautonomous manner, in other words, where PITP $\alpha$ function is not required in neurons but must be present in nonneuronal support cells that nourish neurons. Alternatively, PITP $\alpha$ could exert its functions in a cell autonomous manner where protein activity is required in the neurons themselves. The available information suggests both mechanisms may be relevant. With regard to cell nonautonomous mechanisms of PITP $\alpha$ action, cells overexpressing PITP $\alpha$ are reported to secrete an as yet uncharacterized trophic factor that promotes neuronal survival ex vivo [167]. Presumably, genetic ablation of PITP $\alpha$ function interferes with production of this factor, thereby contributing to neuronal fragility in PITP $\alpha$-deficient animals. A chemical identification of such PITP $\alpha$-regulated trophic factors would constitute an important advance in our understanding of how PITP $\alpha$ helps confer neuroprotection to the spinocerebellar system. A cell nonautonomous mechanism of this nature forecasts that targeted ablation of PITP $\alpha$ function in neuronal support cells, such as glia and/or oligodendrocytes, will recapitulate at least some features of the spinocerebellar inflammatory disease recorded for $p i t p \alpha^{0 / 0}$ mice.

\section{PITPa \& cell autonomous signaling}

PITP $\alpha$ is identified as an essential component in promoting signaling of plasma membranelocalized receptor tyrosine kinases that register extracellular signals and transmit the information to downstream effector pathways. Two such circuits feature prominently in discussions of intracellular mechanisms for PITP $\alpha$ function. First, PITP $\alpha$ was purified as a 
cytosolic factor required for EGFR signaling in a system where the ligand-dependent EGFR stimulation of PLC $\gamma$ was reconstituted in permeabilized cells [153]. In this system, PITP $\alpha$ is posited to deliver PtdIns to the signaling plasma membrane so that a phosphoinositide pool required for forward EGFR signaling is generated by PtdIns 4-OH and PtdIns-4-phosphate 5-OH kinases. Second, in an analogous mechanism, PITP $\alpha$ is reported to be obligatorily required for signaling via the netrin receptor DCC [152]. Netrins are secreted guidance cues that promote axon elongation and direct pathfinding during neuronal development and are essential for the proper formation of major commissures in the brain and spinal chord $[168,169]$. In both studies, the major conclusion is that PITP $\alpha$ binds activated receptor and, in this fashion, brings PtdIns from the ER to the site of receptor-ligand engagement. This supply activity is then posited to stimulate local production of phosphoinositide and, in turn, downstream signaling $[152,153]$. In a related scenario, PITP $\alpha$ is reported as fueling a phosphoinositide-3-OH kinase signaling pathway required for elongation of cortical neurons on specific extracellular matrices ex vivo [170].

The parallel logic proposed for how PITP $\alpha$ promotes EGFR and DCC signaling via cell autonomous mechanisms is attractive because it makes strong predictions regarding how null cells should behave in a physiological context. From the perspective of neuronal development, loss of an amplifying factor such as PITP $\alpha$ should impair spinal cord and brain structures whose development is netrin- or DCC-dependent. Some properties of the pitpa $\alpha^{0 / 0}$ mouse are superficially consistent with such a model $[157,158]$. It is a tenable hypothesis that comprehensive defects in axon guidance would lead to extensive neuronal cell death by apoptosis and, in the case of a hypoglycemic animal, aponecrosis, as is observed in pitpa $\alpha^{0 / 0}$ mice. However, there are discrepancies between the central predictions of the proposed model for functional coupling of PITP $\alpha$ with DCC and in vivo experimental results. Significant defects in netrin signaling result in obvious structural abnormalities of the brain, including deranged development of the anterior and hippocampal commissures, and the corpus callosum [169]. However, the pitp $\alpha 0 / 0$ brain does not present such obvious derangements and, moreover, the pitp $\alpha 0 / 0$ cortex is not significantly smaller than its wildtype counterpart [157]. The idea that PITP $\alpha$ obligatorily promotes DCC signaling is also inconsistent with the properties of the kanga mouse, a mutant animal with a spontaneous and clean deletion of the essential PITP $\alpha$-binding domain in the DCC cytosolic tail $[152,171]$. While this mouse exhibits an abnormal gait, and the kanga brain recapitulates the structural defects observed in netrin-deficient brain, the mouse nonetheless survives to adulthood and is fertile [171]. By contrast, DCC null mice present embryonic lethal phenotypes [168]. The kanga and pitpa $\alpha^{O / 0}$ phenotypes are not consistent with a strong compromise of DCC function in the absence of interaction with PITP $\alpha$.

The EGFR-PITP $\alpha$ forward signaling paradigm formulated from permeabilized cell studies also fails to translate cleanly to authentic physiological contexts. As an active EGFR signaling network initiates a transcriptional response that promotes cellular survival and proliferation [172], loss of a factor that increases the gain on forward-signaling (i.e., PITP $\alpha$ ) should compromise cell vigor and proliferative capacity. However, pitp $\alpha^{0 / 0}$ murine embryonic stem cells retain their tumorigenicity when introduced into nude mice. This is not an outcome obviously consistent with overt growth factor signaling defects. Moreover, PITP $\alpha$ hypomorphic animals do not present obvious waved phenotypes that result from defective hair follicle development in mice with even partial defects in EGFR signaling $[155,157,173]$. The corresponding null phenotypes differ as well. EGFR nullizygosity generally results in embryonic lethality. In some genetic backgrounds, the knockout mice are born alive, but expire within the first postnatal week, and exhibit multiple symptoms of delayed epithelial development. Those 'escapers' show defective eyelid development, deranged terminal differentiation of the epidermis and hair follicles, and loss of structural integrity of intestine - as evidenced by shortening of the organ, reduced numbers of villi and 
hemorrhage. Moreover, EGFR deficiency leads to respiratory failure as a consequence of structurally immature alveoli $[174,175]$. No such defects are reported for $p i t p \alpha^{0 / 0}$ mice.

In summary, the in vivo data are not consistent with simple models invoking obligatory roles for PITP $\alpha$ in forward DCC or EGFR signaling. It remains formally possible that a PITP $\alpha$ involvement in promoting DCC or EGFR signaling is subtle, or that this requirement exhibits an unexpected, and as yet unidentified, tissue-specificity. The idea that significant compensatory mechanisms are engaged in the face of chronic PITP $\alpha$ deficiency cannot yet be dismissed. However, the counter view that PITP $\alpha$ does not promote forward EGFR or DCC signaling must be considered as well. These issues frame a set of important questions for future analysis.

\section{PITPa insufficiencies \& chylomicron retention disease}

A major phenotype associated with pitp $\alpha^{0 / 0}$ homozygosity is failure of such neonates to thrive, in other words, mutant animals achieve only a half to a third of the mass of $P I T P \alpha^{+/+}$ and $P I T P \alpha^{0 /+}$ heterozygous littermates within the first postnatal week. The phenotype is manifested even though the null animals nurse reasonably effectively - as evidenced by direct observation of the act and analysis of stomach contents, and by the fact that pitpo ${ }^{0 / 0}$ nullizygotes are not dehydrated. Whole-body chemical analyses demonstrate the reduced body mass is substantially accounted for by a pathologically low body fat content, a deficiency confirmed by the virtual absence of axillary and inguinal fat pads in nullizygotes [158]. These homeostatic pathologies stem from inefficient processing of dietary fat by the nullizygous animals, and available data report that the homeostatic deficiencies are manifestations of functional derangements in $p i t p \alpha^{0 / 0}$ intestine and liver.

Two lines of evidence indicate that $p i t p \alpha^{0 / 0}$ neonates cannot effectively absorb dietary fat across the intestinal epithelium. First, enterocytes of the $p i t p \alpha^{0 / 0}$ duodenum stain unusually heavily with lipophilic agents (Figure 7) and this property disappears upon prolonged fasting. Second, electron microscopy reveals dramatic accumulations of lipid bodies in the lumen of the enterocyte ER of $p i t p \alpha^{0 / 0}$ neonates - an accretion accompanied by dilations of smooth ER (Figure 7). These accumulated lipid bodies resemble maturing chylomicrons, in effect the lipoprotein transport units that ferry dietary fat via the enterocyte secretory pathway for discharge into the circulation and disbursement throughout the body. Taken together, the morphological data indicate that $p i t p \alpha^{0 / 0}$ enterocytes are competent for the hydrolysis of dietary triglycerides (TGs) into fatty acids and monoacylglycerols, the transport of these hydrolytic products into the enterocyte ER lumen and the reconstitution of these products into TGs for subsequent packaging into what we loosely term as chylomicron precursors. The point of failure is in inefficient export of chylomicron precursors from the enterocyte ER, via the secretory pathway, and into the circulation.

Consistent with this basic scenario, pitp $\alpha^{0 / 0}$ neonates present dramatic reductions in levels of circulating postprandial TG and brain $\alpha$-tocopherol (another lipophilic molecule whose transport across the enterocyte into the circulation requires a functional chylomicron pathway). The collective data suggest a PITP $\alpha$ involvement in the packaging of TG cores into functional chylomicron carriers for transport from the enterocyte ER. Defects in this process hallmark CRD. The threshold requirement for PITP $\alpha$ in chylomicron transport is low given that an $80 \%$ reduction in PITP $\alpha$ expression is insufficient to induce CRD in mice, but a more than $90 \%$ reduction in PITP $\alpha$ load does [157]. The defect is also tissueautonomous as reconstitution of intestine-specific PITP $\alpha$ expression in the null animal rescues the CRD [157].

PITP $\alpha$ involvements in ER functions are unanticipated, as all discussions of functional mechanisms focus on roles for PITP $\alpha$ in modulating plasma membrane signaling circuits 
(see previous sections). It remains to be established whether PITP $\alpha$ is directly, or more indirectly, involved in chylomicron biogenesis. Indirect models would include regulation of chylomicron maturation from a remote compartment via some PITP $\alpha$-dependent signaling pathway. Some concepts for how PITP $\alpha$ may directly interface with chylomicron trafficking from the ER are suggested by recent studies on the etiology of human CRD, such as Anderson's disease and hyperbetalipoproteinemia. These CRD syndromes are autosomal recessive disorders characterized by fat malabsorption and pediatric failure to thrive [176]. The associated human duodenal steatosis, when coupled with other aspects of human CRD symptomology, is similar to the CRD and associated phenotypes of pitpa $\alpha^{0 / 0}$ mice. Positional cloning analyses demonstrate that one mechanism for inherited human CRD stems from autosomal recessive loss-of-function mutations in the SARA2 GTPase [177]. SARA2 is the product of the $S A R I B$ gene, and represents one of the two members of the Sar1-like small GTPases expressed in humans.

The Sar1-like GTPases are conserved from yeast to man and these are essential for nucleation of COPII vesicle formation at organized regions of the ER membrane surface termed ER exit sites [178]. The specific requirement for SARA2/Sar1b in chylomicron packaging for transport from the enterocyte ER is interesting because these lipoprotein cargos are much larger than typical COPII transport vesicles. One interpretation of the genetic data is that SARA2/Sar1b is a privileged GTPase dedicated to formation of atypical COPII vesicles designed to carry unusually large cargos. It follows that PITP $\alpha$ is a similarly privileged component of such a specialized COPII vesicle biogenic pathway [158]. Perhaps PITP $\alpha$ regulates an ER pool of PtdIns-4-phosphate required for biogenesis of a subclass of COPII vesicles dedicated to transport of mega-cargos such as chylomicrons. This proposal is supported by the demonstration that mice expressing wild-type amounts of a PtdIns-bindingdefective mutant of PITP $\alpha$ as the sole source of the protein are indistinguishable from $p i t p \alpha^{0 / 0}$ animals, including with regard to severity of CRD [157]. While the concept that PITP $\alpha$ modulates an ER pool of phosphoinositide departs from dogmatic views that phosphoinositides are localized exclusively to intracellular compartments in the distal secretory pathway [179], recent findings that Sar1-mediated ER exit site formation is regulated by PtdIns-4-phosphate bolster this idea [180].

\section{pitpa $^{0 / 0}$ mice \& hepatic steatosis}

The intestine and liver deploy similar strategies for the processing and export of lipoprotein cargos from the ER into distal compartments of the secretory pathway and, ultimately, into the circulation. In that regard, the $p i t p \alpha^{0 / 0}$ liver also presents extensive microvesicular steatosis as evidenced by the unusually enhanced staining with osmium or the lipophilic dye Oil Red O (Figure 7) [158]. Electron microscopic and lipidomic analyses confirm dramatic intracellular accretion of neutral lipid in the organ. However, in this case, a large fraction of the lipid accumulates in cytosolic lipid droplets and, remarkably, in lipid droplets that populate the nuclear matrix of $p i t p \alpha^{0 / 0}$ hepatocytes (Figure 7) [158]. While $v b / v b$ mice do not present such dramatic symptoms of hepatic steatosis, lipidomic analyses report that the livers of these animals also exhibit elevated levels of neutral lipid [181].

The intrahepatic lipid accumulation is not the result of elevated lipid biosynthesis; the expression of key fatty acid and lipid biosynthetic enzymes is not enhanced. Whether the $p i t p \alpha^{0 / 0}$ liver is defective in lipoprotein trafficking from the ER, as is the case in intestine, remains to be investigated. However, there is no doubt that an important aspect of lipid homeostasis is deranged in $p i t p \alpha^{0 / 0}$ liver, and this deficiency has interesting consequences for outcomes of intestine-specific reconstitution strategies directed at alleviating CRD. Rescue of CRD by intestine-specific expression of PITP $\alpha$, in otherwise pitp $\alpha^{0 / 0}$ mice, levies surprisingly modest improvements in the systemic TG and fat storage defects that characterize this animal. The basis for the inefficient translation of a functionally 
reconstituted intestine to more normal circulating lipoprotein levels appears to rest with enhanced accretion of lipid in the $p i t p \alpha^{0 / 0}$ liver of such animals. We presently consider the amplified accretion to reflect enhanced lipid flow into a dysfunctional liver that cannot adequately process circulating lipoproteins [157]. The increased import is presumably driven by a now functional intestine that efficiently secretes chylomicrons into the circulation of the otherwise $p i t p \alpha^{0 / 0}$ mouse.

\section{PITPa: is there a link between Ins nutrition \& lipoprotein metabolism}

There is an old literature associating nutritional deprivation of Ins with defective lipid clearance from rodent liver and intestine. Rats fed a fatty diet accumulate triacylglycerides and cholesterol in the liver when myoinositol is withheld from the diet [182-184]. This accumulation of neutral lipid is the result of defective mobilization of hepatic TGs [185,186]. In the Mongolian gerbil model, myoinositol starvation resulted in accumulation of lipid in the small intestine [187] - a phenotype associated with qualitative changes in the fatty acid composition of enteric phospholipids $[188,189]$. Notably, this condition was also marked by significantly decreased levels of circulating lipoprotein [190] - a deficit that was established as the consequence of inefficient transport of lipid across the intestine [191].

While these observations were taken as evidence for an involvement of PtdIns metabolism with lipid transport across the intestine [191], no underlying mechanism for the Ins effect has yet been described. The intestinal and hepatic steatosis that characterizes $p i t p \alpha^{0 / 0}$ mice broadly recapitulates the effects of inositol deprivation in rodents. It is now an attractive proposition that Ins deprivation and functional ablation of PITP $\alpha$ share an underlying mechanism for provoking intestinal, and perhaps hepatic, steatosis. In other words, phosphoinositides play an important role in the packaging of unusual cargoes such as chylomicrons, and other lipoprotein particles for transport from the ER to late stages of the secretory pathway. This hypothesis raises the interesting possibility that PITP $\alpha$ sets the efficiency for lipoprotein assembly in intestine and liver and therefore determines the capacity for lipid clearance in liver and intestine.

\section{PITPa \& the pancreas}

The third signature pathology associated with $p i t p \alpha^{0 / 0}$ mice is a severe hypoglycemia where circulating glucose (and insulin) levels are nearly an order of magnitude lower than those of wild-type siblings $[157,158]$. One major defect appears to be in hepatic gluconeogenesis with severe deficits in both proglucagon gene expression and in circulating glucagon levels. As a result, the pitp $\alpha^{0 / 0}$ liver inappropriately stores glycogen in the face of a catastrophic hypoglycemia [158]. As in the case of the CRD, the threshold requirement for PITP $\alpha$ in maintenance of proper glucose homeostasis is low. Functional PITP $\alpha$ reductions of $90 \%$ or greater are required for manifestation of hypoglycemia [157].

The glucose and gluconeogenic derangements on display in $p i t p \alpha^{0 / 0}$ neonates are accompanied by obvious structural derangements of the pancreas. While $p i t p \alpha^{0 / 0}$ exocrine pancreas is morphologically normal, the endocrine pancreas is not. The number of recognizable islets per $p i t p \alpha^{0 / 0}$ pancreas is strongly reduced relative to wild-type, vacuolations are evident in the islets, and the $p i t p \alpha^{0 / 0}$ islet cells themselves are shrunken [158]. It is not yet known whether these pancreatic deficits are the result of indirect damage inflicted by a hostile physiological environment (e.g., associated with CRD), or whether these are manifestations of some other intrinsic developmental problem.

\section{Zebrafish type 1 PITPs}

Zebrafish (Danio rerio) express a mammalian-like cohort of type 1 PITPs with the addition of a unique PITP $\beta$-like protein designated PITP $\gamma$ [37]. The conservation of Type 1 PITP 
roster further extends to interesting details of type 1 PITP diversity; in other words, zebrafish execute precisely the same exon-skipping event as mammals do in generating the canonical and alternative PITP $\beta$ splice variants. As a result, this nonmammalian model is a facile system for study of vertebrate type 1 PITPs, and it is particularly informative with respect to the physiological functions of PITP $\beta$ isoforms. Whereas functional ablation experiments have, to this point, been uninformative for the PITP $\gamma$ isoform, new insights are forthcoming regarding PITP $\beta$ function in this organism. Zebrafish express PITP $\beta$ splice variants predominantly in the eye, specifically in the synaptic pedicles of retinal double cone cells [37]. Morpholino-based silencing experiments demonstrate PITP $\beta$ splice variant activity is required for the biogenesis and/or maintenance of the double cone photoreceptor cell outer segments. The deficits in double cone cell outer segment biogenesis and structure are also reversible. As effectiveness of PITP $\beta$-directed morpholinos wanes with age of the morphant fish and PITP $\beta$ expression is restored, development of morphologically correct and electrophysiologically functional double cone cells is re-engaged [37].

What functional mechanisms underlie PITP $\beta$ splice variant involvement in zebrafish double cone cell outer segment biogenesis/maintenance? Cone cell outer segments are comprised of an intricate network of membraneous discs/lamellae. These membranes are subject to a vigorous course of self-renewal that involves a high-capacity membrane trafficking program [37,192]. The general localization of PITP $\beta$ splice variants to the TGN suggests a scenario where deficiencies in PITP $\beta$ activity subtly compromise Golgi function in double cone cells, and evoke significant defects in biosynthetic trafficking of opsin into the outer segment. If the normally high rates of membrane turnover in these structures are maintained in the face of reduced incorporation of biosynthetic material, outer segment integrity will be compromised. In mammalian rod cells, the SARA adaptor couples PtdIns-3-P cues to syntaxin t-SNARE activity in potentiation of the vesicle fusion events involved in outer segment membrane disc formation and maintenance [193]. One possibility is that zebrafish PITP $\beta$ splice variants support a similarly privileged phosphoinositide-dependent trafficking pathway in double cone cells.

The zebrafish PITP $\alpha$ studies are surprising in that, unlike in mice, functional ablation of PITP $\alpha$ results in defective gastrulation. The failure occurs at a stage where highly migratory cells of the blastoderm extend and converge to cover the yolk cell surface [37]. As in mice, PtdIns-binding is an essential functional property of PITP $\alpha$ activity in zebrafish development. The lack of functional redundancy between PITP $\alpha$ and PITP $\beta$ isoforms is also clearly evident - PITP $\beta$ does not compensate for PITP $\alpha$ deficits in zebrafish development [37]. Thus, different vertebrates employ paralogous type 1 PITPs in substantially different ways.

\section{Conclusion \& future perspective}

The appropriate spatial and temporal regulation of lipid metabolic flux is central to cellular homeostasis - even subtle derangements of this system lead to disease. However, we are far from understanding how diverse territories of the cellular lipid metabolome are interfaced and how lipid metabolic processes are coordinated with lipid signaling. It is becoming increasingly clear that Sec14-like PITPs, and likely PITPs in general, contribute to the integration of diverse aspects of lipid metabolism with phosphoinositide signaling. Physical pictures of how this may happen are emerging, particularly for Sec14-like proteins and these models identify new areas for experimental inquiry. Two general areas are ripe for investigation. First, it will be interesting to discern whether the PtdIns-binding barcodes of Sec14-like proteins not annotated as PITPs (e.g., caytaxin, CRALBP1 and $\alpha$-TTP) forecast authentic inositol-lipid binding capabilities. If so, and it seems likely that it will be so in some cases, the activities of these proteins, and the etiologies of the associated diseases, will 
need to be re-interpreted. Second, the question of PtdIns-presentation function will be particularly interesting from the standpoint of Sec14- or PITP-like modules in complex multidomain proteins. The concept that such PITP-like domains prime production of local phosphoinositide signals in response to metabolic cues, and that these phosphoinositide signals in turn modulate catalytic activities of these complex proteins, describes new conceptual frameworks for how such proteins operate. We expect that Sec14-domains in particular will garner more attention in this regard and will become increasingly attractive targets for pharmacological intervention. The importance of being able to visualize, in living cells, when and where PITPs execute lipid exchange is also clear. Reliable conformational biosensors will be invaluable tools in those efforts and will provide unique approaches towards faithfully imaging PITP-regulated interfaces between lipid metabolism and signaling in vivo.

Finally, we forecast interest in PITPs, and perhaps primarily Sec14-like PITPs, as targets for pharmaceutical intervention in the context of infectious diseases. While not discussed in this review, eukaryotic pathogens express PITP-like proteins - some of which are clearly PITPs. These proteins are sufficiently diverged from their vertebrate paralogs to suggest that identification of specific small molecule inhibitors will be feasible. It is in this area that PITP-directed therapies, and a detailed understanding of PITP biology and biochemistry, may ultimately make the greatest impact in human health.

\section{Executive summary}

\section{Phosphatidylinositol transfer proteins \& inositol signaling}

- Emerging data implicate phosphatidylinositol transfer proteins (PITPs) as instructors of phosphatidylinositol metabolism, a role that contributes to the functional diversification of PI signaling.

- PITPs separate into two evolutionarily unrelated classes: the highly conserved Sec14-like PITPs and the START-domain PITPs.

- Analysis of PITP function has been limited by the inability to describe PITPs using traditional enzyme definitions.

- Structural and genetic studies on the yeast Sec14 have implicated its role as a 'nanoreactor', wherein its primary function is not simple lipid exchange between membranes, but integration of PtdCho metabolism with presentation of PtdIns to PtdIns 4-OH kinase.

\section{Sec14-like PITPs in human disease}

- Sec14-like proteins can form standalone proteins or be part of more complicated landscapes within multidomain proteins.

- Malfunctions in Sec14-like domains result in a variety of human disorders including ataxia with vitamin E deficiency, cayman-type cerebellar ataxia, visual cycle defects, retinitis punctata albescens and neurofibromatosis type 1 .

- Sec14-like proteins, in general, have a conserved PtdIns-binding 'barcode'. Mutations within this region often result in protein dysfunction and are well represented in physiologically relevant mutations.

\section{Physiology of mammalian type I PITPs}

- The mammalian type 1 PITPs are structurally unrelated to the Sec14-like PITPs, but may nonetheless function as nanoreactors. 
- Mice with graded reductions of PITP $\alpha$ expression have been used to dissect the physiological roles of PITP $\alpha$. PITP $\alpha$ deficiency results in spinocerebellar disease, enteric and hepatic steatosis, and hypoglycemia.

- PITP $\alpha$ is implicated in both cell autonomous and cell nonautonomous signaling mechanisms.

- The roles of type 1 PITPs are being addressed in the zebrafish model system, describing a role for PITP $\beta$ in maintaining outer segment integrity in specific cone cells.

\section{Acknowledgments}

The authors thank Jim Alb, Carl Mousley and Lora Yanagisawa for helpful discussions regarding the issues highlighted in this article and for critical reading of the manuscript. The authors are particularly grateful to the quality of the referees' critical input on this work during the review process. Their comments and suggestions greatly improved the manuscript.

This work was supported by grants GM44530, NS37723 and GM081774 from the NIH to Vytas A Bankaitis.

\section{Bibliography}

Papers of special note have been highlighted as:

-of interest

- of considerable interest

1. Michell RH. Inositol derivatives: evolution and functions. Nat. Rev. Mol. Biol. 2008; 9:151-161.

2. Irivine RF. Inositide evolution - towards turtle domination? J. Physiol. 2005; 566:295-300. [PubMed: 15860522]

3. Fruman DA, Meyers RE, Cantley LC. Phosphoinositide kinases. Annu. Rev. Biochem. 1998; 67:481-507. [PubMed: 9759495]

4. Di Paolo G, De Camilli P. Phosphoinositides in cell regulation and membrane dynamics. Nature. 2006; 443:651-657. [PubMed: 17035995]

5. Strahl T, Thorner J. Synthesis and function of membrane phosphoinositides in budding yeast, Saccharomyces cerevisiae. Biochim. Biophys. Acta. 2007; 1771:353-404. [PubMed: 17382260]

6. Majerus PW, York JD. Phosphoinositide phosphatases and disease. J. Lipid Res. 2009; 50:S249S254. [PubMed: 19001665]

7. Liu Y, Bankaitis VA. Phosphoinositide phosphatases in cell biology and disease. Prog. Lipid Res. 2010; 49:201-217. [PubMed: 20043944]

8. Lemmon MA. Membrane recognition by phospholipid-binding domains. Nat. Rev. Mol. Cell. Biol. 2008; 9:99-111. [PubMed: 18216767]

9. Balla T. Inositol-lipid binding motifs: signal integrators through protein-lipid and protein-protein interactions. J. Cell Sci. 2005; 118:2093-2104. [PubMed: 15890985]

10. Mclaughlin S, Murray D. Plasma membrane phosphoinositide organization by protein electrostatics. Nature. 2005; 438:605-611. [PubMed: 16319880]

11. Routt SM, Ryan MM, Tyeryar K, et al. Nonclassical PITPs activate PLD via the Stt4p PtdIns-4kinase and modulate function of late stages of exocytosis in vegetative yeast. Traffic. 2005; 6:1157-1172. [PubMed: 16262726]

12. Schaaf G, Ortlund EA, Tyeryar KR, et al. Functional anatomy of phospholipid binding and regulation of phosphoinositide homeostasis by proteins of the Sec14 superfamily. Mol. Cell. 2008; 29:191-206. [PubMed: 18243114] • High-resolution structural studies establish the strikingly different binding poses for PtdIns and PtdCho in the Sec14 hydrophobic pocket, and describes a body of evidence in support of Sec14-like phosphatidylinositol transfer proteins (PITPs) serving as primeable PtdIns presentation units. The PtdIns- and PtdCho-binding barcodes are also defined. 
13. Bankaitis VA, Mousley CJ, Schaaf G. The Sec14 superfamily and mechanisms for crosstalk between lipid metabolism and lipid signaling. Trends Biochem. Sci. 2010; 35:150-160. [PubMed: 19926291]

14. Wirtz KWA. Phospholipid transfer proteins. Annu. Rev. Biochem. 1991; 60:73-99. [PubMed: 1883207]

15. Michell RH. Inositol phospholipids and cell surface receptor function. Biochim. Biophys. Acta. 1975; 415:81-147. [PubMed: 164246]

16. Li X, Routt SM, Xie Z, et al. Identification of a novel family of nonclassic yeast phosphatidylinositol transfer proteins whose function modulates phospholipase D activity and Sec14p-independent cell growth. Mol. Biol. Cell. 2000; 11:1989-2005. [PubMed: 10848624]

17. Phillips SE, Vincent P, Rizzieri KE, et al. The diverse biological functions of phosphatidylinositol transfer proteins in eukaryotes. Crit. Rev. Biochem. Mol. Biol. 2006; 41:21-49. [PubMed: 16455519]

18. Bankaitis VA, Malehorn DE, Emr SD, Greene R. The Saccharomyces cerevisiae SEC14 gene encodes a cytosolic factor that is required for transport of secretory proteins from the yeast Golgi complex. J. Cell Biol. 1989; 108:1271-1281. [PubMed: 2466847]

19. Bankaitis VA, Aitken JR, Cleves AE, Dowhan W. An essential role for a phospholipid transfer protein in yeast Golgi function. Nature. 1990; 347:561-562. [PubMed: 2215682]

20. Yoder MD, Thomas LM, Tremblay JM, et al. Structure of a multifunctional protein: mammalian phosphatidylinositol transfer protein complexed with phosphatidylcholine. J. Biol. Chem. 2001; 276:9246-9252. [PubMed: 11104777]

21. Milligan SC, Alb JG, Elagina RB, Bankaitis VA, Hyde DR. The phosphatidylinositol transfer protein domain of Drosophila retinal degeneration B protein is essential for photoreceptor cell survival and recovery from light stimulation. J. Cell Biol. 1997; 139:351-363. [PubMed: 9334340]

22. Mousley CJ, Tyeryar K, Ile KE, et al. Trans-Golgi network and endosome dynamics connect ceramide homeostasis with regulation of the unfolded protein response and TOR signaling in yeast. Mol. Biol. Cell. 2008; 19:4785-4803. [PubMed: 18753406]

23. Cleves AE, Novick PJ, Bankaitis VA. Mutations in the $S A C 1$ gene suppress defects in yeast Golgi and yeast actin function. J. Cell Biol. 1989; 109:2939-2950. [PubMed: 2687291]

24. Cleves AE, Mcgee T, Bankaitis V. Phospholipid transfer proteins: a biological debut. Trends Cell Biol. 1991; 1:30-34. [PubMed: 14731807]

25. Cleves AE, Mcgee TP, Whitters EA, et al. Mutations in the CDP-choline pathway for phospholipid biosynthesis bypass the requirement for an essential phospholipid transfer protein. Cell. 1991; 64:789-800. [PubMed: 1997207]

26. Fang M, Kearns BG, Gedvilaite A, et al. Kes1p shares homology with human oxysterol binding protein and participates in a novel regulatory pathway for yeast Golgi-derived transport vesicle biogenesis. EMBO J. 1996; 15:6447-6459. [PubMed: 8978672]

27. Rivas MP, Kearns BG, Xie Z, et al. Pleiotropic alterations in lipid metabolism in yeast sac1 mutants: relationship to 'bypass Sec14p' and inositol auxotrophy. Mol. Biol. Cell. 1999; 10:22352250. [PubMed: 10397762]

28. Li X, Rivas MP, Fang M, et al. Analysis of oxysterol binding protein homologue Kes1p function in regulation of Sec14p-dependent protein transport from the yeast Golgi complex. J. Cell Biol. 2002; 157:63-77. [PubMed: 11916983]

29. Sha B, Phillips SE, Bankaitis VA, Luo M. Crystal structure of the Saccharomyces cerevisiae phosphatidylinositol-transfer protein. Nature. 1998; 391:506-510. [PubMed: 9461221]

30. Phillips SE, Sha B, Topalof L, et al. Yeast Sec14p deficient in phosphatidylinositol transfer activity is functional in vivo. Mol. Cell. 1999; 4:187-197. [PubMed: 10488334]

31. Smirnova TI, Chadwick TG, Voinov MA, et al. Local polarity and hydrogen bonding inside the Sec14p phospholipid-binding cavity: high-field multi-frequency electron paramagnetic resonance studies. Biophys. J. 2007; 92:3686-3695. [PubMed: 17325006]

32. Ryan MM, Temple BRS, Phillips SE, Bankaitis VA. Conformational dynamics of the major yeast phosphatidylinositol transfer protein Sec14p: insight into the mechanisms of phospholipid exchange and diseases of Sec14p-like protein deficiencies. Mol. Biol. Cell. 2007; 18:1928-1942. [PubMed: 17344474] 
33. Wu W-I, Voelker DR. Biochemistry and genetics of interorganelle aminoglycerophospholipid transport. Semin. Cell Dev. Biol. 2002; 13:185-195. [PubMed: 12137739]

34. Holthuis JCM, Levine TP. Lipid traffic: floppy drives and a superhighway. Nat. Rev. Mol. Cell. Biol. 2005; 6:209-220. [PubMed: 15738987]

35. Skinner HB, Alb JG Jr, Whitters EA, Helmkamp GM Jr, Bankaitis VA. Phospholipid transfer activity is relevant to but not sufficient for the essential function of the yeast SEC14 gene product. EMBO J. 1993; 12:4775-4784. [PubMed: 8223486]

36. Tanaka S, Hosaka K. Cloning of a cDNA encoding a second phosphatidylinositol transfer protein of rat brain by complementation of the yeast sec14 mutation. J. Biochem. 1994; 115:981-984. [PubMed: 7961615]

37. Ile KE, Kassen S, Cao C, et al. Zebrafish class 1 phosphatidylinositol transfer proteins: PITP $\beta$ and double cone cell outer segment integrity in retina. Traffic. 2010; 11:1151-1167. [PubMed: 20545905] - PITP $\alpha$ isoforms are shown to play surprisingly specific roles in the establishment and/ or maintenance of opsin-containing outer segments in double cone cells of the fish retina, while PITP $\alpha$ plays essential roles in early embryonic development. The functional nonredundancy of the highly related PITP $\alpha$ and PITP $\alpha$ proteins is clearly demonstrated. Evidence that type 1 PITPs are capable of functioning as PtdIns presentation units is also reported.

38. Mariotti C, Gellera C, Rimoldi M, et al. Ataxia with isolated vitamin E deficiency: neurological phenotype, clinical follow-up and novel mutations in TTPA gene in Italian families. Neurol. Sci. 2004; 25:130-137. [PubMed: 15300460]

39. Aparicio JM, Belanger-Quintana A, Suarez L, et al. Ataxia with isolated vitamin E deficiency: case report and review of the literature. J. Pediatr. Gastroenterol. Nutr. 2001; 33:206-210. [PubMed: 11568526]

40. Doria-Lamba L, De Grandis E, Cristiani E, et al. Efficacious vitamin E treatment in a child with ataxia with isolated vitamin E deficiency. Eur. J. Pediatr. 2006; 165:494-495. [PubMed: 16491382]

41. Yokota T, Igarashi K, Uchihara T, et al. Delayed-onset ataxia in mice lacking $\alpha$-tocopherol transfer protein: model for neuronal degeneration caused by chronic oxidative stress. Proc. Natl Acad. Sci. USA. 2001; 98:15185-15190. [PubMed: 11752462]

42. Terasawa Y, Ladha Z, Leonard SW, et al. Increased atherosclerosis in hyperlipidemic mice deficient in $\alpha$-tocopherol transfer protein and vitamin E. Proc. Natl Acad. Sci. USA. 2000; 97:13830-13834. [PubMed: 11095717]

43. Leonard SW, Terasawa Y, Farese RV Jr, Traber MG. Incorporation of deuterated RRR- or all-rac$\alpha$-tocopherol in plasma and tissues of $\alpha$-tocopherol transfer protein-null mice. Am. J. Clin. Nutr. 2002; 75:555-560. [PubMed: 11864863]

44. Di Donato I, Bianchi S, Federico A. Ataxia with vitamin E deficiency: update of molecular diagnosis. Neurol. Sci. 2010; 31(4):511-515. [PubMed: 20464573]

45. Morley S, Panagabko C, Shineman D, et al. Molecular determinants of heritable vitamin E deficiency. Biochemistry. 2004; 43:4143-4149. [PubMed: 15065857]

46. Morley S, Cecchini M, Zhang W, et al. Mechanisms of ligand transfer by the hepatic tocopherol transfer protein. J. Biol. Chem. 2008; 283:17797-17804. [PubMed: 18458085] - Evidence that $\alpha$ tocopherol binding protein ( $\alpha \mathrm{TTP}$ ) binding to membranes is enhanced by vitamin $\mathrm{E}$ is described. That some ataxia with vitamin E deficiency (AVED)-associated missense substitutions blunt the effects of vitamin $\mathrm{E}$ on membrane binding by $\alpha \mathrm{TTP}$ is also demonstrated.

47. Qian J, Atkinson J, Manor D. Biochemical consequences of heritable mutations in the $\alpha$-tocopherol transfer protein. Biochemistry. 2006; 45:8236-8242. [PubMed: 16819822] - $\alpha$ TTP is shown to facilitate vitamin E secretion from cells, and that AVED versions of the protein compromise delivery of vitamin $\mathrm{E}$ from endosomal/lysosomal compartments to the plasma membrane. The authors posit that $\alpha$ TTP regulates vitamin E packaging into transport vesicles in endosomal compartments for delivery to the plasma membrane.

48. Akihiro H, Makoto A, Yuji S, et al. Affinity for $\alpha$-tocopherol transfer protein as a determinant of the biological activities of vitamin E analogs. FEBS Lett. 1997; 409:105-108. [PubMed: 9199513]

49. Panagabko C, Morley S, Hernandez M, et al. Ligand specificity in the CRAL-TRIO protein family. Biochemistry. 2003; 42:6467-6474. [PubMed: 12767229] 
50. Zhang W, Frahm G, Morley S, Manor D, Atkinson J. Effect of bilayer phospholipid composition and curvature on ligand transfer by the $\alpha$-tocopherol transfer protein. Lipids. 2009; 44:631-641. [PubMed: 19458973] • Using FRET-based assays, $\alpha$ TTP is shown to use a collisional mechanism for tocopherol transfer, and that increased membrane curvature, but not anionic phospholipid content, enhance $\alpha$ TTP association with membranes. The authors conclude that the hydrophobic properties of $\alpha$ TTP dominate the interaction of $\alpha$ TTP with membranes.

51. Traber MG. Vitamin E regulatory mechanisms. Annu. Rev. Nutr. 2007; 27:347-362. [PubMed: 17439363]

52. Clarke MW, Burnett JR, Croft KD. Vitamin E in human health and disease. Crit. Rev. Clin. Lab. Sci. 2008; 45:417-450. [PubMed: 18712629]

53. Horiguchi M, Arita M, Kaempf-Rotzoll DE, et al. $\mathrm{pH}$-dependent translocation of $\alpha$-tocopherol transfer protein ( $\alpha$-TTP) between hepatic cytosol and late endosomes. Genes Cells. 2003; 8:789800. [PubMed: 14531858]

54. Qian J, Morley S, Wilson K, et al. Intracellular trafficking of vitamin E in hepatocytes: the role of tocopherol transfer protein. J. Lipid Res. 2005; 46:2072-2082. [PubMed: 16024914]

55. Arita M, Nomura K, Arai H, Inoue K. $\alpha$-Tocopherol transfer protein stimulates the secretion of $\alpha$ tocopherol from a cultured liver cell line through a brefeldin A-insensitive pathway. Proc. Natl Acad. Sci. USA. 1997; 94:12437-12441. [PubMed: 9356467]

56. Bomar JM, Benke PJ, Slattery EL, et al. Mutations in a novel gene encoding a CRAL-TRIO domain cause human Cayman ataxia and ataxia/dystonia in the jittery mouse. Nat. Genet. 2003; 35:264-269. [PubMed: 14556008]

57. Nystuen A, Benke PJ, Merren J, Stone EM, Sheffield VC. A cerebellar ataxia locus identified by DNA pooling to search for linkage disequilibrium in an isolated population from the Cayman Islands. Hum. Mol. Genet. 1996; 5:525-531. [PubMed: 8845847]

58. Xiao J, Gong S, Ledoux MS. Caytaxin deficiency disrupts signaling pathways in cerebellar cortex. Neuroscience. 2007; 144:439-461. [PubMed: 17092653] - Transcriptomic profiling experiments implicate deranged PtdIns signaling as a contributing factor to the neuropathologies of the dystonic rat. The collective data indicate caytaxin is important for the Purkinje cells response to climbing fiber input in cerebellar cortex.

59. Xiao J, Ledoux MS. Caytaxin deficiency causes generalized dystonia in rats. Mol. Brain Res. 2005; 141:181-192. [PubMed: 16246457]

60. Ledoux MS, Lorden JF. Abnormal spontaneous and harmaline-stimulated Purkinje cell activity in the awake genetically dystonic rat. Exp. Brain Res. 2002; 145:457-467. [PubMed: 12172657] • Electrophysiological experiments show Purkinje cells fire at normal rates in the dystonic rat, and that firing abnormalities reflect defects in the interface between the inferior olive to climbing fiber synapses on Purkinje cells.

61. Martins-De-Souza D, Gattaz W, Schmitt A, et al. Prefrontal cortex shotgun proteome analysis reveals altered calcium homeostasis and immune system imbalance in schizophrenia. Eur. Arch. Psychiatry. Clin. Neurosci. 2009; 259:151-163. [PubMed: 19165527]

62. Ledoux MS, Lorden JF, Ervin JM. Cerebellectomy eliminates the motor syndrome of the genetically dystonic rat. Exp. Neurol. 1993; 120:302-310. [PubMed: 8491286]

63. Ledoux MS, Lorden JF, Meinzen-Derr J. Selective elimination of cerebellar output in the genetically dystonic rat. Brain Res. 1995; 697:91-103. [PubMed: 8593599]

64. Raike RS, Jinnah HA, Hess EJ. Animal models of generalized dystonia. NeuroRx. 2005; 2:504512. [PubMed: 16389314]

65. Grelle G, Kostka S, Otto A, et al. Identification of VCP/p97, carboxyl terminus of Hsp70interacting protein (CHIP), and amphiphysin II Interaction partners using membrane-based human proteome arrays. Mol. Cell Proteomics. 2006; 5:234-244. [PubMed: 16275660]

66. Buschdorf JP, Chew LL, Soh UJK, Liou Y-C, Low BC. Nerve growth factor stimulates interaction of cayman ataxia protein BNIP-H/Caytaxin with peptidyl-prolyl isomerase Pin1 in differentiating neurons. PLoS One. 2008; 3:E2686. [PubMed: 18628984]

67. Hayakawa Y, Itoh M, Yamada A, Mitsuda T, Nakagawa T. Expression and localization of Cayman ataxia-related protein, Caytaxin, is regulated in a developmental- and spatial-dependent manner. Brain Res. 2007; 1129:100-109. [PubMed: 17157273] 
68. Aoyama T, Hata S, Nakao T, et al. Cayman ataxia protein caytaxin is transported by kinesin along neurites through binding to kinesin light chains. J. Cell Sci. 2009; 122:4177-4185. [PubMed: 19861499]

69. Travis GH, Golczak M, Moise AR, Palczewski K. Diseases caused by defects in the visual cycle: retinoids as potential therapeutic agents. Annu. Rev. Pharmacol. Toxicol. 2007; 47:469-512. [PubMed: 16968212]

70. Thompson DA, Gal A. Vitamin A metabolism in the retinal pigment epithelium: genes, mutations, and diseases. Prog. Retin. Eye. Res. 2003; 22:683-703. [PubMed: 12892646]

71. Saari JC, Crabb JW. Focus on molecules: cellular retinaldehyde-binding protein (CRALBP). Exp. Eye Res. 2005; 81:245-246. [PubMed: 16085009]

72. Saari JC, Nawrot M, Kennedy BN, et al. Visual cycle impairment in cellular retinaldehyde binding protein (CRALBP) knockout mice results in delayed dark adaptation. Neuron. 2001; 29:739-748. [PubMed: 11301032]

73. Stecher H, Gelb MH, Saari JC, Palczewski K. Preferential release of 11-cis-retinol from retinal pigment epithelial cells in the presence of cellular retinaldehyde-binding protein. J. Biol. Chem. 1999; 274:8577-8585. [PubMed: 10085092]

74. Saari JC, Bredberg DL, Noy N. Control of substrate flow at a branch in the visual cycle. Biochemistry. 1994; 33:3106-3112. [PubMed: 8130225]

75. Nawrot M, West K, Huang J, et al. Cellular retinaldehyde-binding protein interacts with ERMbinding phosphoprotein 50 in retinal pigment epithelium. Invest. Ophthalmol. Vis. Sci. 2004; 45:393-401. [PubMed: 14744877]

76. Saari JC, Nawrot M, Stenkamp RE, Teller DC, Garwin GG. Release of 11-cis-retinal from cellular retinaldehyde-binding protein by acidic lipids. Mol. Vis. 2009; 15:844-854. [PubMed: 19390642] - Acidic phospholipids are shown to stimulate retinal release from cellular retinal-binding protein (CRALBP). This discovery identifies the first factors that may physiologically regulate retinal release from CRALBP.

77. Golovleva I, Bhattacharya S, Wu Z, et al. Disease-causing mutations in the cellular retinaldehyde binding protein tighten and abolish ligand interactions. J. Biol. Chem. 2003; 278:12397-12402. [PubMed: 12536144] - Either ablation or enhancement of retinoid binding is found to disturb CRALBP function in the visual cycle. These results suggest a balance of retinoid binding and release (i.e., appropriately robust retinoid exchange) is essential for the biological function of CRALBP.

78. Maw MA, Kennedy B, Knight A, et al. Mutation of the gene encoding cellular retinaldehydebinding protein in autosomal recessive retinitis pigmentosa. Nat. Genet. 1997; 17:198-200. [PubMed: 9326942] - Molecular pedigree analyses of nonsyndromic autosomal recessive retinitis pigmentosa identify an arginine to glutamine missense substitution at residue 150 of CRALBP. Biochemical studies show that this substitution ablates 11-cis retinaldehyde binding by CRALBP.

79. Golovleva I, Köhn L, Burstedt M, Daiger S, Sandgren O. Mutation spectra in autosomal dominant and recessive retinitis pigmentosa in Northern Sweden. Adv. Exp. Med. Biol. 2010; 664:255-262. [PubMed: 20238024]

80. Burstedt M, Sandgren O, Holmgren G, Forsman-Semb K. Bothnia dystrophy caused by mutations in the cellular retinaldehyde-binding protein gene (RLBP1) on chromosome 15q26. Invest. Ophthalmol. Vis. Sci. 1999; 40:995-1000. [PubMed: 10102298]

81. He X, Lobsiger J, Stocker A. Bothnia dystrophy is caused by domino-like rearrangements in cellular retinaldehyde-binding protein mutant R234W. Proc. Natl Acad. Sci. USA. 2009; 106:18545-18550. [PubMed: 19846785] - The crystal structures of human CRALBP and the R234W Bothnia dystrophy variant complexed with 11-cis-retinal are reported. R234 is found to reside in a basic surface cleft, and the $\mathrm{R} 234 \mathrm{~W}$ missense substitution is proposed to compromise membrane docking and to encourage tighter binding of 11-cis-retinal by CRALBP.

82. Deeg CA, Raith AJ, Amann B, et al. CRALBP is a highly prevalent autoantigen for human autoimmune uveitis. Clin. Dev. Immunol. 2007; 2007:39245. [PubMed: 18317528]

83. Treadwell JA, Pagniello KB, Singh SM. Genetic segregation of brain gene expression identifies retinaldehyde binding protein 1 and syntaxin 12 as potential contributors to ethanol preference in mice. Behav. Genet. 2004; 34:425-439. [PubMed: 15082940] 
84. Curwin AJ, Mcmaster CR. Structure and function of the enigmatic Sec14 domain-containing proteins and the etiology of human disease. Future Lipidol. 2008; 3:399-410.

85. Zhang HM, Cheung P, Yanagawa B, Mcmanus BM, Yang DC. BNips: a group of proapoptotic proteins in the Bcl-2 family. Apoptosis. 2003; 8:229-236. [PubMed: 12766483]

86. García-Mata R, Burridge K. Catching a GEF by its tail. Trends Cell Biol. 2007; 17:36-43. [PubMed: 17126549]

87. Rossman KL, Der CJ, Sondek J. GEF means go: turning on RHO GTPases with guanine nucleotide-exchange factors. Nat. Rev. Mol. Cell. Biol. 2005; 6:167-180. [PubMed: 15688002]

88. Kostenko EV, Mahon GM, Cheng L, Whitehead IP. The Sec14 homology domain regulates the cellular distribution and transforming activity of the Rho-specific guanine nucleotide exchange factor Dbs. J. Biol. Chem. 2005; 280:2807-2817. [PubMed: 15531584] - Evidence is presented to suggest that the Dbs Sec14 domain negatively regulates protein activity by intramolecular interactions with the Dbs $\mathrm{pH}$ domain, and is also required for targeting of Dbs to Golgi membranes.

89. Ueda S, Kataoka T, Satoh T. Role of the Sec14-like domain of Dbl family exchange factors in the regulation of Rho family GTPases in different subcellular sites. Cell. Signal. 2004; 16:899-906. [PubMed: 15157669] - The Sec14 domain of the Dbl family proteins Dbl and Ost is shown to direct the appropriate subcellular localization of these guanine nucleotide exchange proteins (GEFs) and to influence the intracellular distribution of their common substrate Cdc42.

90. Johnson RC, Penzes P, Eipper BA, Mains RE. Isoforms of Kalirin, a neuronal Dbl family member, generated through use of different $5^{\prime}$ - and $3^{\prime}$-ends along with an internal translational initiation site. J. Biol. Chem. 2000; 275:19324-19333. [PubMed: 10777487]

91. Portales-Casamar E, Briançon-Marjollet A, Fromont S, Triboulet R, Debant A. Identification of novel neuronal isoforms of the Rho-GEF Trio. Biol. Cell. 2006; 98:183-193. [PubMed: 16033331]

92. Komai K, Mukae-Sakairi N, Kitagawa M, Shiozawa S. Characterization of novel splicing variants of the mouse MCF-2 (DBL) proto-oncogene. Biochem. Biophys. Res. Commun. 2003; 309:906909. [PubMed: 13679059]

93. Vanni C, Mancini P, Gao Y, et al. Regulation of proto-Dbl by intracellular membrane targeting and protein stability. J. Biol. Chem. 2002; 277:19745-19753. [PubMed: 11907027]

94. Fardin P, Ognibene M, Vanni C, et al. Induction of epithelial mesenchimal transition and vasculogenesis in the lenses of Dbl oncogene transgenic mice. PLoS One. 2009; 4:E7058. [PubMed: 19759912]

95. Hirsch E, Pozzato M, Vercelli A, et al. Defective dendrite elongation but normal fertility in mice lacking the Rho-Like GTPase activator Dbl. Mol. Cell. Biol. 2002; 22:3140-3148. [PubMed: 11940671]

96. Mcpherson CE, Eipper BA, Mains RE. Genomic organization and differential expression of Kalirin isoforms. Gene. 2002; 284:41-51. [PubMed: 11891045]

97. Rabiner CA, Mains RE, Eipper BA. Kalirin: a dual Rho guanine nucleotide exchange factor that is so much more than the sum of its many parts. Neuroscientist. 2005; 11:148-160. [PubMed: 15746383]

98. Schiller MR, Ferraro F, Wang Y, et al. Autonomous functions for the Sec14p/spectrin-repeat region of Kalirin. Exp. Cell Res. 2008; 314:2674-2691. [PubMed: 18585704] - The Kalirin Sec14like domain is shown to bind PtdIns-3,5- $\mathrm{P}_{2}$ and PtdIns-3-P and to contribute to the ability of the protein to regulate cell morphology in a manner independent of its GEF activities.

99. Penzes P, Johnson RC, Sattler R, et al. The neuronal Rho-GEF Kalirin-7 interacts with PDZ domain containing proteins and regulates dendritic morphogenesis. Neuron. 2001; 29:229-242. [PubMed: 11182094]

100. Cahill ME, Xie Z, Day M, et al. Kalirin regulates cortical spine morphogenesis and diseaserelated behavioral phenotypes. Proc. Natl Acad. Sci. USA. 2009; 106:13058-13063. [PubMed: 19625617]

101. Xie Z, Cahill ME, Penzes P. Kalirin loss results in cortical morphological alterations. Mol. Cell Neurosci. 2010; 43:81-89. [PubMed: 19800004]

102. Krug T, Manso H, Gouveia L, et al. Kalirin: a novel genetic risk factor for ischemic stroke. Hum. Genet. 2010; 127:513-523. [PubMed: 20107840] 
103. Wang L, Hauser ER, Shah SH, et al. Peakwide mapping on chromosome 3q13 identifies the kalirin gene as a novel candidate gene for coronary artery disease. Am. J. Hum. Genet. 2007; 80:650-663. [PubMed: 17357071]

104. Youn H, Ji I, Ji HP, Markesbery WR, Ji TH. Under-expression of Kalirin-7 increases iNOS activity in cultured cells and correlates to elevated iNOS activity in Alzheimer's disease hippocampus. J. Alzheimers Dis. 2007; 12:271-281. [PubMed: 18057561]

105. Hayashi-Takagi A, Takaki M, Graziane N, et al. Disrupted-in-Schizophrenia 1 (DISC1) regulates spines of the glutamate synapse via Rac1. Nat. Neurosci. 2010; 13:327-332. [PubMed: 20139976]

106. Penzes P, Jones KA. Dendritic spine dynamics - a key role for kalirin-7. Trends Neurosci. 2008; 31:419-427. [PubMed: 18597863]

107. Saneyoshi T, Fortin DA, Soderling TR. Regulation of spine and synapse formation by activitydependent intracellular signaling pathways. Curr. Opin. Neurobiol. 2010; 20:108-115. [PubMed: 19896363]

108. Xie Z, Srivastava DP, Photowala H, et al. Kalirin-7 controls activity-dependent structural and functional plasticity of dendritic spines. Neuron. 2007; 56:640-656. [PubMed: 18031682]

109. Nishida K, Kaziro Y, Satoh T. Association of the proto-oncogene product Dbl with G protein $\beta \gamma$ subunits. FEBS Lett. 1999; 459:186-190. [PubMed: 10518015]

110. Briancon-Marjollet A, Ghogha A, Nawabi H, et al. Trio mediates Netrin-1-induced Rac1 activation in axon outgrowth and guidance. Mol. Cell. Biol. 2008; 28:2314-2323. [PubMed: 18212043]

111. O'Brien SP, Seipel K, Medley QG, et al. Skeletal muscle deformity and neuronal disorder in Trio exchange factor-deficient mouse embryos. Proc. Natl Acad. Sci. USA. 2000; 97:12074-12078. [PubMed: 11050238]

112. Zheng M, Simon R, Mirlacher M, et al. TRIO amplification and abundant mRNA expression Is associated with invasive tumor growth and rapid tumor cell proliferation in urinary bladder cancer. Am. J. Pathol. 2004; 165:63-69. [PubMed: 15215162]

113. Chattopadhyay I, Singh A, Phukan R, et al. Genome-wide analysis of chromosomal alterations in patients with esophageal squamous cell carcinoma exposed to tobacco and betel quid from highrisk area in India. Mutat. Res. 2010; 696:130-138. [PubMed: 20083228]

114. Sun Y-J, Nishikawa K, Yuda H, et al. Solo/Trio8, a membrane-associated short isoform of Trio, modulates endosome dynamics and neurite elongation. Mol. Cell. Biol. 2006; 26:6923-6935. [PubMed: 16943433]

115. Estrach S, Schmidt S, Diriong S, et al. The human Rho-GEF Trio and Its target GTPase RhoG are involved in the NGF pathway, leading to neurite outgrowth. Curr. Biol. 2002; 12:307-312. [PubMed: 11864571]

116. Liu Z, Adams HC, Whitehead IP. The Rho-specific guanine nucleotide exchange factor Dbs regulates breast cancer cell migration. J. Biol. Chem. 2009; 284:15771-15780. [PubMed: 19366686]

117. Tcherkezian J, Lamarche-Vane N. Current knowledge of the large RhoGAP family of proteins. Biol. Cell. 2007; 099:67-86. [PubMed: 17222083]

118. Wang L, Yang L, Debidda M, Witte D, Zheng Y. Cdc42 GTPase-activating protein deficiency promotes genomic instability and premature aging-like phenotypes. Proc. Natl Acad. Sci. USA. 2007; 104:1248-1253. [PubMed: 17227869]

119. Wang L, Yang L, Burns K, Kuan C-Y, Zheng Y. Cdc42GAP regulates c-Jun N-terminal kinase (JNK)-mediated apoptosis and cell number during mammalian perinatal growth. Proc. Natl Acad. Sci. USA. 2005; 102:13484-13489. [PubMed: 16157885]

120. Yang L, Wang L, Zheng Y. Gene targeting of Cdc42 and Cdc42GAP affirms the critical involvement of $\mathrm{Cdc} 42$ in filopodia induction, directed migration, and proliferation in primary mouse embryonic fibroblasts. Mol. Biol. Cell. 2006; 17:4675-4685. [PubMed: 16914516]

121. Hatjiharissi E, Ngo H, Leontovich AA, et al. Proteomic analysis of waldenstrom macroglobulinemia. Cancer Res. 2007; 67:3777-3784. [PubMed: 17440091] 
122. Jin L, Liu G, Zhang C-H, et al. Nm23-H1 regulates the proliferation and differentiation of the human chronic myeloid leukemia K562 cell line: a functional proteomics study. Life Sci. 2009; 84:458-467. [PubMed: 19302816]

123. Engelse M, Laurens N, Verloop R, Koolwijk P, Van Hinsbergh V. Differential gene expression analysis of tubule forming and non-tubule forming endothelial cells: CDC42GAP as a counterregulator in tubule formation. Angiogenesis. 2008; 11:153-167. [PubMed: 18060510]

124. Sirokmány G, Szidonya L, Káldi K, et al. Sec14 homology domain targets p50RhoGAP to endosomes and provides a link between Rab and Rho GTPases. J. Biol. Chem. 2006; 281:60966105. [PubMed: 16380373] - The Sec14-like domain of p50RhoGAP is demonstrated to be required for localization of this protein to endosomes, and for its interaction with the endosomal ras-like GTPase Rab11. The authors propose that p50RhoGAP is an interface through which Rab and Rho GTPase activity is coordinated in control of receptor-mediated endocytosis, and that Sec14 domains may regulate protein-protein interactions.

125. Moskwa P, Paclet M-H, Dagher M-C, Ligeti E. Autoinhibition of p50 Rho GTPase-activating protein (GAP) is released by prenylated small GTPases. J. Biol. Chem. 2005; 280:6716-6720. [PubMed: 15596440]

126. Cichowski K, Jacks T. NF1 tumor suppressor gene function: narrowing the GAP. Cell. 2001; 104:593-604. [PubMed: 11239415]

127. D’ Angelo I, Welti S, Bonneau F, Scheffzek K. A novel bipartite phospholipid-binding module in the neurofibromatosis type 1 protein. EMBO Rep. 2006; 7:174-179. [PubMed: 16397625] -. Crystallographic analyses identify a novel Sec14/PH-like module in neurofibromin that binds phospholipids. The unusual domain architecture suggests a detailed model for how the PH-like domain might regulate phospholipid binding by the Sec14-like domain.

128. Friedman JM. Epidemiology of neurofibromatosis type 1. Am. J. Med. Genet. 1999; 89:1-6. [PubMed: 10469430]

129. Ferner RE, Huson SM, Thomas N, et al. Guidelines for the diagnosis and management of individuals with neurofibromatosis-1. J. Med. Genet. 2007; 44:81-88. [PubMed: 17105749]

130. Gottfried ON, Viskochil DH, Couldwell WT. Neurofibromatosis type 1 and tumorigenesis: molecular mechanisms and therapeutic implications. Neurosurg. Focus. 2010; 28:E8. [PubMed: 20043723]

131. Xu G, O’Connell P, Viskochil D, et al. The neurofibromatosis type 1 gene encodes a protein related to GAP. Cell. 1990; 62:599-608. [PubMed: 2116237]

132. Aravind L, Neuwald AF, Ponting CP. Sec14p-like domains in NF1 and Dbl-like proteins indicate lipid regulation of Ras and Rho signaling. Curr. Biol. 1999; 9:R195-R197. [PubMed: 10209105]

133. Bonneau F, Lenherr ED, Pena V, Hart DJ, Scheffzek K. Solubility survey of fragments of the neurofibromatosis type 1 protein neurofibromin. Protein Expr. Purif. 2009; 65:30-37. [PubMed: 19111619]

134. Fahsold R, Hoffmeyer S, Mischung C, et al. Minor lesion mutational spectrum of the entire NF1 gene does not explain its high mutability but points to a functional domain upstream of the GAPrelated domain. Am. J. Hum. Genet. 2000; 66:790-818. [PubMed: 10712197]

135. Upadhyaya M, Maynard J, Osborn M, et al. Characterisation of germline mutations in the neurofibromatosis type 1 (NF1) gene. J. Med. Genet. 1995; 32:706-710. [PubMed: 8544190]

136. Welti S, Fraterman S, D'angelo I, Wilm M, Scheffzek K. The Sec14 homology module of neurofibromin binds cellular glycerophospholipids: mass spectrometry and structure of a lipid complex. J. Mol. Biol. 2007; 366:551-562. [PubMed: 17187824] -- The Sec14-like domain of neurofibromin is demonstrated to harbor both phospholipid binding and exchange activities. These discoveries support the idea that the Sec14 domain is a lipid-sensing unit that coordinates lipid metabolism with neurofibromin activity.

137. Huynh H, Wang X, Li W, et al. Homotypic secretory vesicle fusion induced by the protein tyrosine phosphatase MEG2 depends on polyphosphoinositides in T cells. J. Immunol. 2003; 171:6661-6671. [PubMed: 14662869]

138. Alonso A, Sasin J, Bottini N, et al. Protein tyrosine phosphatases in the human genome. Cell. 2004; 117:699-711. [PubMed: 15186772] 
139. Saito K, Tautz L, Mustelin T. The lipid-binding SEC14 domain. Biochim. Biophys. Acta. 2007; 1771:719-726. [PubMed: 17428729]

140. Kruger JM, Fukushima T, Cherepanov V, et al. Protein-tyrosine phosphatase MEG2 is expressed by human neutrophils. J. Biol. Chem. 2002; 277:2620-2628. [PubMed: 11711529]

141. Zhao R, Fu X, Li Q, Krantz SB, Zhao ZJ. Tyrosine phosphatase MEG2 modulates murine development and platelet and lymphocyte activation through secretory vesicle function. J. Exp. Med. 2005; 202:1587-1597. [PubMed: 16330817]

142. Huynh H, Bottini N, Williams S, et al. Control of vesicle fusion by a tyrosine phosphatase. Nat. Cell Biol. 2004; 6:831-839. [PubMed: 15322554]

143. Saito K, Williams S, Bulankina A, Höning S, Mustelin T. Association of protein-tyrosine phosphatase MEG2 via its Sec14p homology domain with vesicle-trafficking proteins. J. Biol. Chem. 2007; 282:15170-15178. [PubMed: 17387180] - The MEG2 Sec14-domain is shown to be required for targeting of this enzyme to secretory vesicles, and for mediating functionally relevant MEG2 interactions with TIP47 and Arfaptin2.

144. Phillips SE, Ile KE, Boukhelifa M, Huijbregts RPH, Bankaitis VA. Specific and nonspecific membrane-binding determinants cooperate in targeting phosphatidylinositol transfer protein $\beta$ isoform to the mammalian trans-Golgi network. Mol. Biol. Cell. 2006; 17:2498-2512. [PubMed: 16540520]

145. Morgan CP, Allen-Baume V, Radulovic M, et al. Differential expression of a C-terminal splice variant of phosphatidylinositol transfer protein $\beta$ lacking the constitutive-phosphorylated Ser 262 that localizes to the Golgi compartment. Biochem. J. 2006; 398:411-421. [PubMed: 16780419]

146. Schouten A, Agianian B, Westerman J, et al. Structure of apo-phosphatidylinositol transfer protein- $\alpha$ provides insight into membrane association. EMBO J. 2002; 21:2117-2121. [PubMed: 11980708]

147. Tilley SJ, Skippen A, Murray-Rust J, et al. Structure-function analysis of phosphatidylinositol transfer protein A bound to human phosphatidylinositol. Structure. 2004; 12:317-326. [PubMed: 14962392]

148. Alb JG Jr, Phillips SE, Rostand K, et al. Genetic ablation of phosphatidylinositol transfer protein function in murine embryonic stem cells. Mol. Biol. Cell. 2002; 13:739-754. [PubMed: 11907258]

149. Hay JC, Martin TF. Phosphatidylinositol transfer protein required for ATP-dependent priming of $\mathrm{Ca}^{2+}$-activated secretion. Nature. 1995; 374:173-177. [PubMed: 7877690]

150. Ohashi M, Jan De Vries K, Frank R, et al. A role for phosphatidylinositol transfer protein in secretory vesicle formation. Nature. 1995; 377:544-547. [PubMed: 7566155]

151. Thomas GMH, Cunningham E, Fensome A, et al. An essential role for phosphatidylinositol transfer protein in phospholipase C-mediated inositol lipid signaling. Cell. 1993; 74:919-928. [PubMed: 8374957]

152. Xie Y, Ding YQ, Hong Y, et al. Phosphatidylinositol transfer protein- $\alpha$ in netrin-1-induced PLC signalling and neurite outgrowth. Nat. Cell Biol. 2005; 7:1124-1132. [PubMed: 16244667] • PITP $\alpha$ is reported to bind the C-terminal tail of the netrin receptor deleted in colorectal cancer (DCC) and describes evidence interpreted as demonstrating an essential involvement of PITP $\alpha$ in DCC signaling.

153. Kauffmann-Zeh A, Thomas G, Ball A. Requirement for phosphatidylinositol transfer protein in epidermal growth factor signaling. Science. 1995; 268:1188-1190. [PubMed: 7761838]

154. Carvou N, Holic R, Li M, et al. Phosphatidylinositol- and phosphatidylcholine-transfer activity of PITP $\beta$ is essential for COPI-mediated retrograde transport from the Golgi to the endoplasmic reticulum. J. Cell Sci. 2010; 123:1262-1273. [PubMed: 20332109] - A role for PITP $\alpha$ in regulating Golgi morphology, and in facilitating production of a phospshoinositide pool, important for retrograde membrane trafficking from the Golgi complex to the endoplasmic reticulum (ER)/intermediate compartment, is reported. Nuclear envelope dysmorphologies in PITP $\alpha$-depleted cells are also noted.

155. Weimar WR, Lane PW, Sidman RL. Vibrator (vb): a spinocerebellar system degeneration with autosomal recessive inheritance in mice. Brain Res. 1982; 251:357-364. [PubMed: 7139332] 
156. Hamilton BA, Smith DJ, Mueller KL, et al. The vibrator mutation causes neurodegeneration via reduced expression of PITP $\alpha$ : positional complementation cloning and extragenic suppression. Neuron. 1997; 18:711-722. [PubMed: 9182797]

157. Alb JG, Phillips SE, Wilfley LR, Philpot BD, Bankaitis VA. The pathologies associated with functional titration of phosphatidylinositol transfer protein $\alpha$ activity in mice. J. Lipid Res. 2007; 48:1857-1872. [PubMed: 17525475] - Transgenic mouse experiments demonstrate that PtdInsbinding is an essential activity required for biological function of PITP $\alpha$. Electrophysiological data also demonstrate that PITP $\alpha$ plays no obvious roles in synaptic function, but is required in a cell-autonomous fashion, for chylomicron biogenesis in enterocytes.

158. Alb JGJ, Cortese JD, Phillips SE, et al. Mice lacking phosphatidylinositol transfer protein- $\alpha$ exhibit spinocerebellar degeneration, intestinal and hepatic steatosis, and hypoglycemia. J. Biol. Chem. 2003; 278:33501-33518. [PubMed: 12788952]

159. Nyquist DA, Helmkamp GMJ. Developmental patterns in rat brain of phosphatidylinositol synthetic enzymes and phosphatidylinositol transfer protein. Biochim. Biophys. Acta. 1989; 987:165-170. [PubMed: 2557926]

160. Utsunomiya A, Owada Y, Yoshimoto T, Kondo H. Localization of gene expression for phosphatidylinositol transfer protein in the brain of developing and mature rats. Mol. Brain Res. 1997; 45:349-352. [PubMed: 9149113]

161. Imai H, Tanaka S, Fukusato T, Yamashita S, Hosaka K. Differential distribution of mRNAs encoding phosphatidylinositol transfer proteins $\alpha$ and $\beta$ in the central nervous system of the rat. Mol. Brain Res. 1997; 46:256-264. [PubMed: 9191100]

162. Floyd JA, Gold DA, Concepcion D, et al. A natural allele of Nxf1 suppresses retrovirus insertional mutations. Nat. Genet. 2003; 35:221-228. [PubMed: 14517553]

163. Elble RJ. Central mechanisms of tremor. J. Clin. Neurophysiol. 1996; 13:133-144. [PubMed: 8849968]

164. Gauthier S, Sniderman A. Action tremor as a manifestation of chylomicron retention disease. Ann. Neurol. 1983; 14:591. [PubMed: 6651243]

165. Trapp BD, Nave K-A. Multiple sclerosis: an immune or neurodegenerative disorder? Ann. Rev. Neurosci. 2008; 31:247-269. [PubMed: 18558855]

166. Schwab C, Mcgeer PL. Inflammatory aspects of Alzheimer disease and other neurodegenerative disorders. J. Alzheimers Dis. 2008; 13:359-369. [PubMed: 18487845]

167. Bunte H, Schenning M, Sodaar P, et al. A phosphatidylinositol transfer protein $\alpha$-dependent survival factor protects cultured primary neurons against serum deprivation-induced cell death. J. Neurochem. 2006; 97:707-715. [PubMed: 16573656]

168. Fazeli A, Dickinson SL, Hermiston ML, et al. Phenotype of mice lacking functional Deleted in colorectal cancer (Dcc) gene. Nature. 1997; 386:796-804. [PubMed: 9126737]

169. Serafini T, Colamarino SA, Leonardo ED, et al. Netrin-1 Is required for commissural axon guidance in the developing vertebrate nervous system. Cell. 1996; 87:1001-1014. [PubMed: 8978605]

170. Cosker KE, Shadan S, Van Diepen M, et al. Regulation of PI3K signalling by the phosphatidylinositol transfer protein PITP $\alpha$ during axonal extension in hippocampal neurons. J. Cell Sci. 2008; 121:796-803. [PubMed: 18285448] - PITP $\alpha$ is reported to be required for laminin-dependent extension of axonal processes in hippocampal neurons on the basis of its ability to promote localized stimulation of PtdIns 3-OH kinase activity.

171. Finger JH, Bronson RT, Harris B, et al. The netrin 1 receptors Unc5h3 and Dcc are necessary at multiple choice points for the guidance of corticospinal tract axons. J. Neurosci. 2002; 22:1034610356. [PubMed: 12451134]

172. Jones SM, Kazlauskas A. Growth factor-dependent signaling and cell cycle progression. Chem. Rev. 2001; 101:2413-2424. [PubMed: 11749380]

173. Luetteke NC, Phillips HK, Qiu TH, et al. The mouse waved-2 phenotype results from a point mutation in the EGF receptor tyrosine kinase. Genes Dev. 1994; 8:399-413. [PubMed: 8125255]

174. Miettinen PJ, Berger JE, Meneses J, et al. Epithelial immaturity and multiorgan failure in mice lacking epidermal growth factor receptor. Nature. 1995; 376:337-341. [PubMed: 7630400] 
175. Sibilia M, Wagner EF. Strain-dependent epithelial defects in mice lacking the EGF receptor. Science. 1995; 269:234-238. [PubMed: 7618085]

176. Charcosset M, Sassolas A, Peretti N, et al. Anderson or chylomicron retention disease: molecular impact of five mutations in the SAR1B gene on the structure and the functionality of Sar1b protein. Mol. Genet. Metab. 2008; 93:74-84. [PubMed: 17945526]

177. Jones B, Jones EL, Bonney SA, et al. Mutations in a Sar1 GTPase of COPII vesicles are associated with lipid absorption disorders. Nat. Genet. 2003; 34:29-31. [PubMed: 12692552] Genome-wide linkage and screening analyses show that mutations in SARA2, the Sar1b GTPase structural gene, are associated with several inherited disorders of fat malabsorption. The results suggest a privileged Sarlb-dependent pathway for the packaging and transport of chylomicrons particles from the enterocyte ER to distal compartments of the secretory pathway.

178. Miller EA, Barlowe C. Regulation of coat assembly-sorting things out at the ER. Curr. Opin. Cell Biol. 2001; 22(4):447-453. [PubMed: 20439155]

179. D'angelo G, Vicinanza M, Di Campli A, De Matteis MA. The multiple roles of PtdIns(4)P - not just the precursor of PtdIns(4,5)P2. J. Cell Sci. 2008; 121:1955-1963. [PubMed: 18525025]

180. Blumental-Perry A, Haney CJ, Weixel Kelly M, et al. Phosphatidylinositol 4-phosphate formation at ER exit sites regulates ER export. Dev. Cell. 2006; 11:671-682. [PubMed: 17084359] •PtdIns 4-phosphate is found to promote Sar1-dependent biogenesis of COPII vesicles on the surface of the ER. This phosphoinositide is shown to be actively generated at assembly sites for COPII vesicles where it promotes nucleation of COPII coat assembly at ER 'exit sites'.

181. Monaco ME, Kim J, Ruan W, et al. Lipid metabolism in phosphatidylinositol transfer protein $\alpha$ deficient vibrator mice. Biochem. Biophys. Res. Commun. 2004; 317:444-450. [PubMed: 15063778]

182. Hayashi E, Maeda T, Tomita T. The effect of myo-inositol deficiency on lipid metabolism in rats. I. The alteration of lipid metabolism in myo-inositol deficient rats. Biochim. Biophys. Acta. 1974; 360:134-145. [PubMed: 4479416]

183. Burton LE, Wells WW. Myo-inositol metabolism during lactation and development in the rat. The prevention of lactation-induced fatty liver by dietary myo-inositol. J. Nutr. 1976; 106:16171628. [PubMed: 978267]

184. Burton LE, Wells WW. Characterization of the lactation-dependent fatty liver in myo-inositol deficient rats. J. Nutr. 1977; 107:1871-1883. [PubMed: 578525]

185. Hayashi E, Maeda T, Tomita T. The effect of myo-inositol deficiency on lipid metabolism in rats. II. The mechanism of triacylglycerol accumulation in the liver of myo-inositol-deficient rats. J. Nutr. 1974; 360:146-155.

186. Burton LE, Wells WW. Myo-inositol deficiency: studies on the mechanism of lactationdependent fatty liver formation in the rat. J. Nutr. 1979; 109:1483-1491. [PubMed: 458502]

187. Kroes JF, Hegsted DM, Hayes KC. Inositol deficiency in gerbils: dietary effects on the intestinal lipodystrophy. J. Nutr. 1973; 103:1448-1453. [PubMed: 4126828]

188. Woods MN, Hegsted DM. Quantitative and qualitative changes in phospholipid in the intestine of the gerbil and the development of lipodystrophy. J. Nutr. 1979; 109:2146-2151. [PubMed: 92556]

189. Chu SH, Hegsted DM. Myo-inositol deficiency in gerbils: changes in phospholipid composition of intestinal microsomes. J. Nutr. 1980; 110:1217-1223. [PubMed: 7381591]

190. Chu SH, Hegsted DM. Myo-inositol deficiency in gerbils: comparative study of the intestinal lipodystrophy in Meriones unguiculatus and Meriones libycus. J. Nutr. 1980; 110:1209-1216. [PubMed: 6155458]

191. Chu SW, Geyer RP. Myo-inositol action on gerbil intestine. Association of phosphatidylinositol metabolism with lipid clearance. Biochim. Biophys. Acta. 1982; 710:63-70. [PubMed: 6173076]

192. Young RW. Biogenesis and renewal of visual cell outer segment membranes. Exp. Eye Res. 1974; 18:215-223. [PubMed: 4600243]

193. Chuang JZ, Zhao Y, Sung C-H. SARA-regulated vesicular targeting underlies formation of the light-sensing organelle in mammalian rods. Cell. 2007; 130:535-547. [PubMed: 17693260]

194. Boyd JM, Malstrom S, Subramanian T, et al. Adenovirus E1B $19 \mathrm{kDa}$ and Bcl-2 proteins interact with a common set of cellular proteins. Cell. 1994; 79:341-351. [PubMed: 7954800] 
195. Sall A, Zhang HM, Qiu D, et al. Proapoptotic activity of mBNIP-21 depends on its BNIP-2 and Cdc42GAP homology $(\mathrm{BCH})$ domain and is enhanced by coxsackievirus B3 infection. Cell Microbiol. 2010; 12(5):599-614. [PubMed: 19951366]

196. Zhou YT, Guy GR, Low BC. BNIP-2 induces cell elongation and membrane protrusions by interacting with Cdc42 via a unique Cdc42-binding motif within its BNIP-2 and Cdc42GAP homology domain. Exp. Cell Res. 2005; 303:263-274. [PubMed: 15652341]

197. Qin W, Hu J, Guo M, et al. BNIPL-2, a novel homologue of BNIP-2, interacts with Bcl-2 and Cdc42GAP in apoptosis. Biochem. Biophys. Res. Commun. 2003; 308:379-385. [PubMed: 12901880]

198. Xie L, Qin W, Li J, et al. BNIPL-2 promotes the invasion and metastasis of human hepatocellular carcinoma cells. Oncol. Rep. 2007; 17:605-610. [PubMed: 17273740]

199. Machida T, Fujita T, Ooo ML, et al. Increased expression of proapoptotic BMCC1, a novel gene with the BNIP2 and Cdc42GAP homology $(\mathrm{BCH})$ domain, is associated with favorable prognosis in human neuroblastomas. Oncogene. 2005; 25:1931-1942. [PubMed: 16288218]

200. Clarke RA, Zhao Z, Guo A-Y, et al. New genomic structure for prostate cancer specific gene PCA3 within BMCC1: implications for prostate cancer detection and progression. PLoS ONE. 2009; 4:E4995. [PubMed: 19319183]

201. Valencia CA, Cotten SW, Liu R. Cleavage of BNIP-2 and BNIP-XL by caspases. Biochem. Biophys. Res. Commun. 2007; 364:495-501. [PubMed: 17961507]

202. Soh UJK, Low BC. BNIP2 extra long inhibits RhoA and cellular transformation by Lbc RhoGEF via its BCH domain. J. Cell Sci. 2008; 121:1739-1749. [PubMed: 18445682]

203. Johnstone CN, Castellví-Bel S, Chang LM, et al. ARHGAP8 is a novel member of the RHOGAP family related to ARHGAP1/CDC42GAP/p50RHOGAP: mutation and expression analyses in colorectal and breast cancers. Gene. 2004; 36:59-71. [PubMed: 15225876]

204. Lua BL, Low BC. Activation of EGF receptor endocytosis and ERK1/2 signaling by BPGAP1 requires direct interaction with EEN/endophilin II and a functional RhoGAP domain. J. Cell Sci. 2005; 118:2707-2721. [PubMed: 15944398]

205. Lua BL, Low BC. BPGAP1 interacts with cortactin and facilitates its translocation to cell periphery for enhanced cell migration. Mol. Biol. Cell. 2004; 15:2873-2883. [PubMed: 15064355]

206. Song JY, Lee JK, Lee NW, et al. Microarray analysis of normal cervix, carcinoma in situ, and invasive cervical cancer: identification of candidate genes in pathogenesis of invasion in cervical cancer. Int. J. Gynecol. Cancer. 2008; 18:1051-1059. [PubMed: 18217980]

207. Shang X, Zhou YT, Low BC. Concerted regulation of cell dynamics by BNIP-2 and Cdc42GAP homology/Sec14p-like, proline-rich, and GTPase-activating protein domains of a novel Rho GTPase-activating protein, BPGAP1. J. Biol. Chem. 2003; 278:45903-45914. [PubMed: 12944407]

208. Kong YH, Ye GM, Qu K, et al. Cloning and characterization of a novel, human cellular retinaldehyde-binding protein CRALBP-like (CRALBPL) gene. Biotechnol. Lett. 2006; 28:1327-1333. [PubMed: 16802092]

209. Zhao S, Xu C, Qian H, et al. Cellular retinaldehyde-binding protein-like (CRALBPL), a novel human Sec14p-like gene that is upregulated in human hepatocellular carcinomas, may be used as a marker for human hepatocellular carcinomas. DNA Cell Biol. 2008; 27:159-163. [PubMed: 18271718]

210. Katoh Y, Ritter B, Gaffry T, et al. The Clavesin family, neuron-specific lipid- and clathrinbinding Sec14 proteins regulating lysosomal morphology. J. Biol. Chem. 2009; 284:2764627654. [PubMed: 19651769]

211. Ribeiro FM, Ferreira LT, Marion S, et al. SEC14-like protein 1 interacts with cholinergic transporters. Neurochem. Int. 2007; 50:356-364. [PubMed: 17092608]

212. Wang X, Ni J, Hsu CL, et al. Reduced expression of tocopherol-associated protein (TAP/ Sec14L2) in human breast cancer. Cancer Invest. 2009; 27:971-977. [PubMed: 19909011]

213. Johnykutty S, Tang P, Zhao H, et al. Dual expression of $\alpha$-tocopherol-associated protein and estrogen receptor in normal/benign human breast luminal cells and the downregulation of $\alpha$ - 
tocopherol-associated protein in estrogen-receptor-positive breast carcinomas. Mod. Pathol. 2009; 22:770-775. [PubMed: 19305383]

214. Shibata N, Jishage KI, Arita M, et al. Regulation of hepatic cholesterol synthesis by a novel protein (SPF) that accelerates cholesterol biosynthesis. FASEB J. 2006; 20:2642-2644. [PubMed: 17077281]

215. Ni J, Wen X, Yao J, et al. Tocopherol-associated protein suppresses prostate cancer cell growth by inhibition of the phosphoinositide 3-kinase pathway. Cancer Res. 2005; 65:9807-9816. [PubMed: 16267002]

216. Wen XQ, Li X-J, Su Z-L, et al. Reduced expression of $\alpha$-tocopherol-associated protein is associated with tumor cell proliferation and the increased risk of prostate cancer recurrence. Asian J. Androl. 2007; 9:206-212. [PubMed: 17334589]

217. Kempná P, Zingg J-M, Ricciarelli R, et al. Cloning of novel human SEC14p-like proteins: ligand binding and functional properties. Free Radic. Biol. Med. 2003; 34:1458-1472. [PubMed: 12757856]

218. Bortner JD, Das A, Umstead TM, et al. Down-regulation of 14-13-3 isoforms and annexin A5 proteins in lung adenocarcinoma induced by the tobacco-specific nitrosamine NNK in the A/J mouse revealed by proteomic analysis. J. Proteome Res. 2009; 8:4050-4061. [PubMed: 19563208]

219. Miehe S, Bieberstein A, Arnould I, et al. The phospholipid-binding protein SESTD1 is a novel regulator of the transient receptor potential channels TRPC4 and TRPC5. J. Biol. Chem. 2010; 285:12426-12434. [PubMed: 20164195]

220. Bezzerides VJ, Ramsey IS, Kotecha S, Greka A, Clapham DE. Rapid vesicular translocation and insertion of TRP channels. Nat. Cell Biol. 2004; 6:709-720. [PubMed: 15258588]

221. Hong SK, Levin C, Brown J, et al. Pregastrula expression of zebrafish extraembryonic genes. BMC Develop. Biol. 2010; 10:42.

\section{Website}

301. The Scripps Research Institute - Mutagenetix home page. http://mutagenetix.scripps.edu/home.cfm 


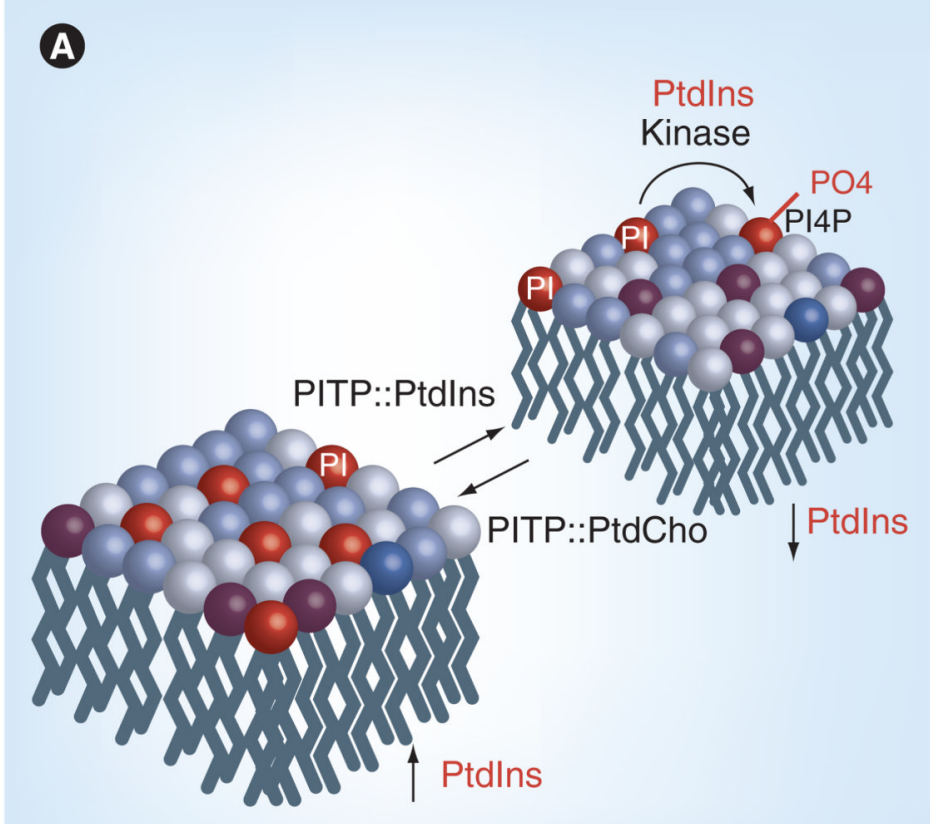

Transfer model
B

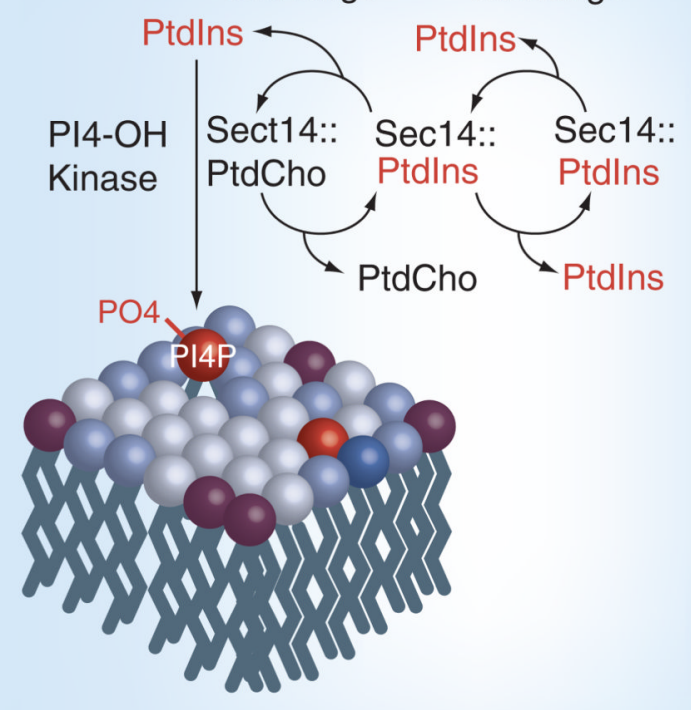

'Nanoreactor' model

Figure 1. Transfer versus nanoreactor models for phosphatidylinositol transfer protein function (A) Lipid transfer models invoke a vectorial carrier function for PITPs where PtdIns is transported from membranes of high PtdIns concentration (endoplasmic reticulum) to relatively PtdIns-poor membranes of the distal compartments of the secretory pathway which house PtdIns-4-OH kinase activity (TGN/endosomes or plasma membrane). These models describe productive transfer as involving one heterotypic exchange reaction per donor and acceptor membrane (i.e., two such exchanges per cycle). (B) The 'nanoreactor' model predicts that PITPs stimulate PI4-OH kinase activity by executing multiple rounds of phospholipid-exchange at a single membrane site. Only heterotypic exchange reactions generate PtdIns configurations suitable for effective PtdIns presentation. PI4P: Phosphatidylinositol-4-phosphate; PITP: Phosphatidylinositol transfer protein; PO4: Phosphate; PtdCho: Phosphatidylcholine; PtdIns: Phosphatidylinositol. 


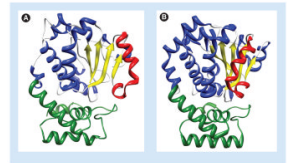

Figure 2. The Sec14 fold

Crystal structure of two Saccharomyces cerevisiae Sec14-like phosphatidylinositol transfer proteins. (A) The major yeast PITP, Sec14 is shown in its open conformation (pdb 1AUA two bound detergent molecules are excluded). (B) The close Sec14 homolog Sfh1 in its closed conformation (pdb 3B7Z - bound phospholipid omitted). The $\beta$-strands comprising the floor of the phospholipid-binding pocket are in yellow, while the $\alpha$-helices that form the walls of the pocket are in blue. Access to the hydrophobic pocket is mediated by conformational transitions of the $\mathrm{A}_{10} / \mathrm{T}_{4}$ 'helical gate' shown in red. The four N-terminal $\alpha$ helices ( $\alpha 1-\alpha 4)$ comprise the N-terminal lobe (or 'tripod motif') (green). 

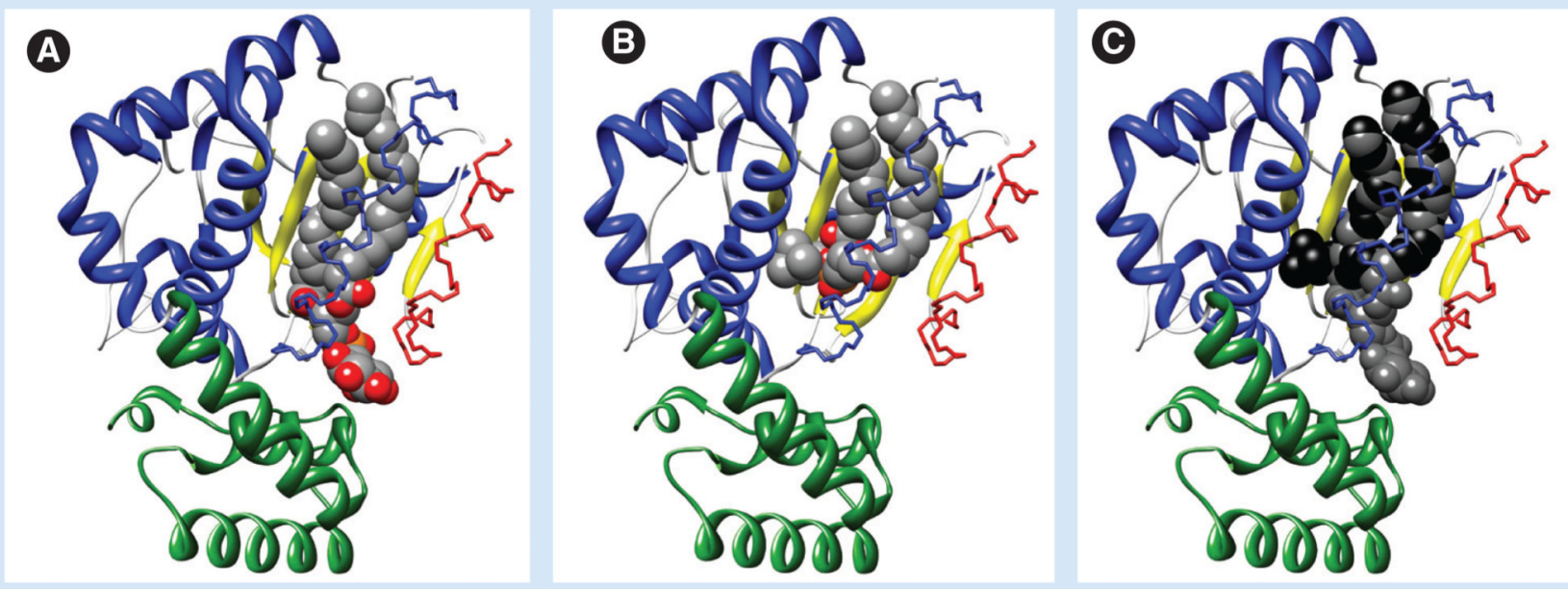

Figure 3. Differential phospholipid-binding strategies by Sec14-like phosphatidylinositol transfer proteins

Structure of Sfh1 bound to: (A) PtdIns (pdb 3B7Z); (B) PtdCho (pdb 3B7Z). (C) A description of the configurations of both phosphatidylinositol (PtdIns; gray) and phosphatidylcholine (PtdCho; black) in the Sfh1/Sec14-fold. The data are from crystals composed of approximately equal numbers of unit cells of Sfh1 bound to PtdIns and Sfh1 bound to PtdCho (pdb 3B7Z). The $\mathrm{A}_{10} / \mathrm{T}_{4}$ 'helical gate' that mediates lipid entry is in red, surrounding $\alpha$-helices are in blue, the 'tripod motif' is in green and $\beta$-strands that compose the floor of the hydrophobic pocket are in yellow. The polar headgroups of PtdIns and PtdCho bind at distinct sites within the hydrophobic pocket, while the acyl chain space within the hydrophobic pocket overlaps for these phospholipids. 


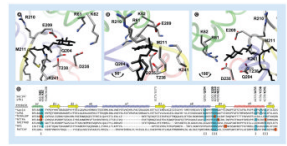

Figure 4. The phosphatidylinositol-binding barcode in Sec14-like proteins

(A) Crystal structure of Sfh1 bound to phosphatidylinositol (PtdIns; black; 3B7N) highlighting residues within the PtdIns binding barcode. The tripod-motif is in green, the floor of the hydrophobic pocket is in yellow, and the $\alpha$-helices are in blue with the exception of the helical gate, which is in red. (B) Orientation of the Sfh1 molecule is rotated by $90^{\circ}$ counterclockwise parallel to the floor. (C) Orientation of the Sfh1 molecule is rotated by $180^{\circ}$ counterclockwise parallel to the floor. (D) ClustalX2 alignments of selected Sec14superfamily members (identified at right; proteins whose crystal structures have been solved are indicated with an '*') were superimposed onto the Sfh1 crystal structure using secondary structural elements as a guide (diagrammed at top). Residues critical for PtdIns headgroup and backbone coordination are boxed and shaded in cyan - I, coordinate the Ins-headgroup; II, coordinate the glycerol backbone; III, coordinate the phosphate moiety through which the Ins headgroup is esterified to the glycerol backbone. Positions of missense substitution within the PtdIns-binding barcode of the corresponding Sec14-like protein that cause disease are highlighted by orange boxes. 
Yeast Sec14-like proteins

$-\mathrm{O}-$

Mammalian Sec14-like proteins
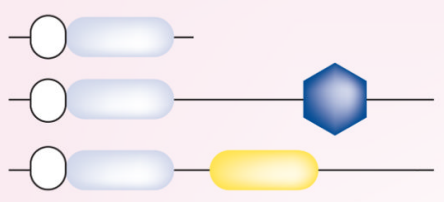

$-1-$
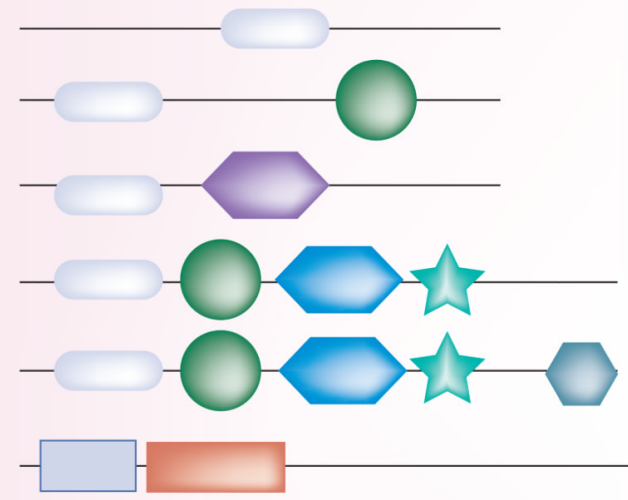

$-$
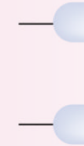

Sec14, Sfh1-Sfh5

CRALBP, CRALBPL, TTPA

PTP-MEG2

SEC14L2, SEC14L3, SEC14L4

SEC14L1, SEC14L5

BNIP-2, BNIP-L, Caytaxin/BINP-H

SESTD1

ARHGAP1/p50RhoGAP/ CDC42GAP/RhoGAP1

DBL/MCF2, MCF2L2

DBS/Ost/MCF2L

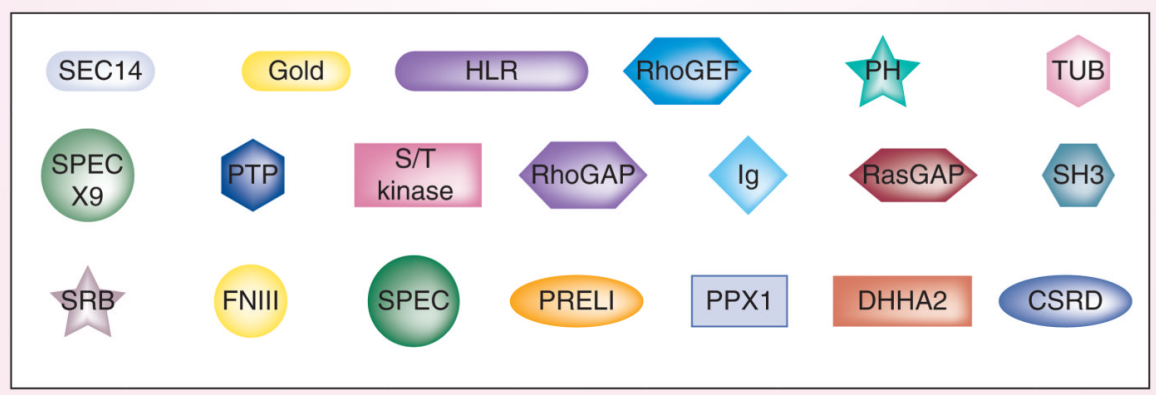

Figure 5. Domain arrangements of Sec14-like proteins

Representative Sec14-like proteins are schematized and ordered by general complexity. Domains of interest are identified. The CRAL-TRIO domain N1 lobe is depicted as a white circle. 

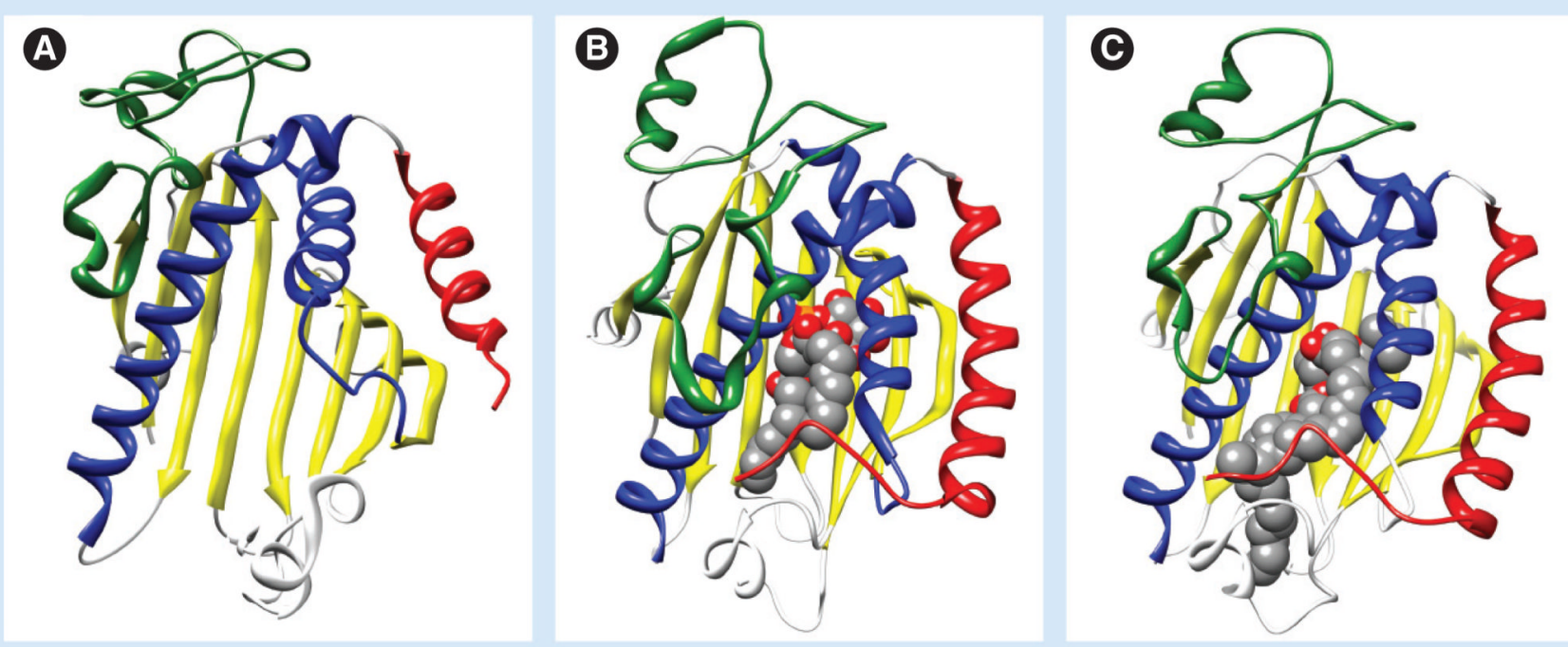

Figure 6. Phosphatidylinositol transfer proteins $\alpha$ structures

(A) Phosphatidylinositol transfer protein (PITP)- $\alpha$ apo-structure depicting an open conformation (pdb 1KCM); (B) PtdIns-bound form (pdb 1UW5); (C) PtdCho-bound form (pdb 1T27). The eight $\beta$-strands (yellow) of PITP $\alpha$ comprise the hydrophobic cavity floor and two $\alpha$-helices generate the cavity walls (blue). Additional components of PITP $\alpha$ include a regulatory loop (green), a C-terminal region (red) and a lipid exchange loop (gray). 


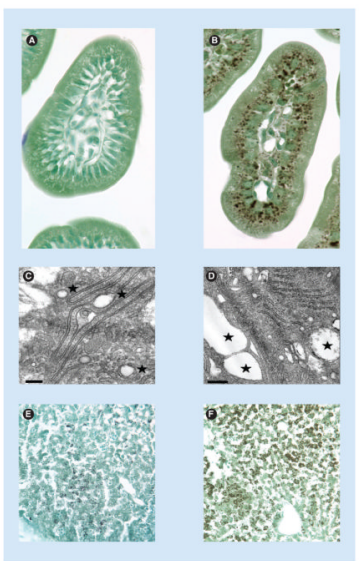

Figure 7. Intestinal and hepatic steatosis in phosphatidylinositol transfer proteins in $\alpha$-deficient mice

Intestinal slices stained for neutral lipid content with osmium from (A) $p i t p \alpha^{+/+}$and (B) pitp $\alpha^{0 / 0}$ mice. Note the obvious accumulation of neutral lipid in mutant enterocytes. This accumulation is dependent on nursing and chases only slowly during periods of fast. The phenotype Is also obvious in electron micrographs of the villi of duodenal enterocytes from (C) pitp $^{+/+}$and (D) pitp $^{0 / 0}$ mice (scale bars are 0.2 and $0.5 \mu \mathrm{M}$, respectively). Lipid deposits are highlighted by asterisks. Liver slices stained with osmium from (E) pitp $\alpha^{+/+}$ and (F) pitp $^{0 / 0}$ mice are also shown. 
Table 1

Other selected Sec14-like proteins.

\begin{tabular}{|lllr|}
\hline Gene & Protein & Description & Ref. \\
\hline BNIP-2 & BNIP-2 & $\begin{array}{l}\text { Proapoptotic and promotes Cdc42-mediated cell elongation through its } \\
\text { Sec14-like domain }\end{array}$ & {$[85,194-196]$} \\
\hline BNIPL & BNIPL/BNIPL2 & Proapoptotic, increases cell migration and may play a role in metastasis & {$[85,197,198]$} \\
\hline BMCC1/BNIP-XL & BMCC1/BNIP-XL & $\begin{array}{l}\text { Proapoptotic protein highly expressed in the human nervous system. } \\
\text { Interacts with RhoA via Sec14 domain and is a favorable signature in } \\
\text { neuroblastomas }\end{array}$ & {$[85,199-202]$} \\
\hline BPGAP1 & BPGAP1 & $\begin{array}{l}\text { Promotes pseudopodia formation, Erk signaling. Elevated levels associated } \\
\text { with colorectal cancer and invasive cervical cancer }\end{array}$ & {$[203-207]$} \\
\hline CRALBPL/RLBP1L1 & Clavesin1 & $\begin{array}{l}\text { Regulates late endosome/lysosome morphology in neurons and is } \\
\text { upregulated in hepatocellular carcinoma }\end{array}$ & {$[208-210]$} \\
\hline Clavesin2 & Regulates late endosome/lysosome morphology in neurons & {$[210]$} \\
\hline Sec14L1 & SEC14L1 & Regulates cholinergic transporters and synaptic vesicle formation & {$[139,211]$} \\
\hline Sec14L5 & SEC14L5 & Unknown & {$[139]$} \\
\hline Sec14L2 & SEC14L2/TAP1/SPF & $\begin{array}{l}\text { Plays a role in cholesterol synthesis during fasting. In humans, a potential } \\
\text { link to breast carcinogenesis and prostate cancer }\end{array}$ & {$[139,212-216]$} \\
\hline Sec14L3 & SEC14L3/TAP2/p45 & Potential link with drug-induced lung adenocarcinoma in mice & {$[139,217,218]$} \\
\hline Sec14L4 & SEC14L4/TAP3 & Unknown & {$[139]$} \\
\hline SESTD1 & Solo/HIPLP & $\begin{array}{l}\text { Elevated levels in the thalamus and brain with potential functions in cone } \\
\text { guidance and smooth muscle contraction }\end{array}$ & {$[219,220,210]$} \\
\hline MOSPD2 & MSPD2 & $\begin{array}{l}\text { Unknown function in humans but is localized with zebrafish maternal } \\
\text { expression in eggs }\end{array}$ & {$[221]$} \\
\hline
\end{tabular}

